ARTICLE

Received 26 Mar 2014 | Accepted 16 Oct 2014 | Published 27 Nov $2014 \quad$ DOl: 10.1038/ncomms6583

\title{
FGF signalling specifies haematopoietic stem cells through its regulation of somitic Notch signalling
}

Yoonsung Lee ${ }^{1}$, Jennifer E. Manegold ${ }^{1}$, Albert D. Kim¹, Claire Pouget ${ }^{1}$, David L. Stachura ${ }^{1,2}$, Wilson K. Clements ${ }^{1,3} \&$ David Traver $^{1}$

Haematopoietic stem cells (HSCs) derive from haemogenic endothelial cells of the primitive dorsal aorta (DA) during vertebrate embryogenesis. The molecular mechanisms governing this unique endothelial to haematopoietic transition remain unclear. Here, we demonstrate a novel requirement for fibroblast growth factor (FGF) signalling in HSC emergence. This requirement is non-cell-autonomous, and acts within the somite to bridge the Wnt and Notch signalling pathways. We previously demonstrated that Wnt16 regulates the somitic expression of two Notch ligands, deltaC ( $d / c)$ and deltaD ( $d l d)$, whose combined function is required for HSC fate. How Wnt16 connects to Notch function has remained an open question. Our current studies demonstrate that FGF signalling, via FGF receptor 4 (Fgfr4), mediates a signaltransduction pathway between Wnt16 and Dlc, but not DId, to regulate HSC specification. Our findings demonstrate that FGF signalling acts as a key molecular relay within the developmental HSC niche to instruct HSC fate.

\footnotetext{
${ }^{1}$ Department of Cellular and Molecular Medicine and Section of Cell and Developmental Biology, University of California, San Diego, La Jolla, California 92093, USA. ${ }^{2}$ Department of Biological Sciences, California State University, Chico, California 95929, USA. ${ }^{3}$ Department of Hematology, St Jude Children's Research Hospital, Memphis, Tennessee 38105, USA. Correspondence and requests for materials should be addressed to D.T. (email: dtraver@ucsd.edu).
} 
$\mathrm{V}$ ertebrate haematopoiesis initiates sequentially with primitive and definitive waves of blood cell production during embryogenesis ${ }^{1-3}$. Of these embryonic blood precursors, only HSCs persist into adulthood and are responsible for lifelong replenishment of the haematopoietic system. In all vertebrate animals studied, HSCs arise from haemogenic endothelium in the floor of the $\mathrm{DA}^{4,5}$. Our current understanding of HSC formation suggests that this endothelial to haematopoietic transition (EHT), which occurs during a limited window in embryonic development, gives rise to the entire pool of HSCs for the life of the organism. A major goal of regenerative medicine is to replicate the development of HSCs in vitro from human pluripotent precursors. Despite decades of effort, this goal has not been achieved. A better understanding of the molecular cues utilized by the embryo to pattern HSCs from mesodermal precursors could inform these approaches.

Development of HSCs requires complex interactions between diverse molecular signalling pathways and downstream intracellular transduction networks. These pathways include Hedgehog signalling, which is required for development of endothelial progenitors and $\mathrm{HSCs}^{6-8}$, vascular endothelial growth factor (Vegf) signalling, which is critical for vasculogenesis and HSC specification $^{9-11}$, bone morphogenetic protein (BMP) signalling, which specifies vascular cells from mesoderm ${ }^{12,13}$, and Notch signalling, which is essential for HSC generation from haemogenic endothelial cells ${ }^{14-16}$. The FGF signalling pathway has likewise been shown to be important in mesoderm formation $^{17,18}$ and vasculogenesis ${ }^{19,20}$, but only a handful of studies have addressed the role of FGF signalling in the development of the haematopoietic lineages.

FGF signalling has been demonstrated to regulate formation of primitive haematopoietic cells by negatively regulating erythroid gene expression in Xenopus ${ }^{21}$. In the avian system, FGFs block primitive erythroid differentiation and promote endothelial development $^{22}$. In contrast, Fgf21 knockdown in zebrafish reduced the formation of erythroid and myeloid cells ${ }^{23}$. In vitro studies indicated that FGFs induced myeloid proliferation in human bone marrow cultures ${ }^{24}$. Although the role of FGF signalling in primitive haematopoiesis has been reasonably well studied, its contribution to definitive HSC formation has never been addressed. Studies of FGF signalling and HSCs in adult mice indicate that long-term repopulating HSCs are found exclusively within an FGFR1-expressing population, and that ectopic provision of FGF1 can stimulate the in vitro expansion of HSCs $^{25}$. However, recent in vivo studies showed that FGFR1 is not required for the homeostasis of adult HSCs but rather in the recovery of haematopoiesis following injury by enhancing HSC proliferation $^{26}$.

In this study, we utilized transgenic zebrafish in which FGF signalling can be inducibly blocked ${ }^{27}$. Loss of FGF signalling during early somitogenesis stages led to a loss of HSCs without disrupting development of primitive haematopoiesis or endothelium. During the temporal knockdown window, the FGF target genes pea3 and erm, as well as the receptors fgfr 1 and $f g f r 4$, were expressed in somites but not in posterior lateral mesoderm (PLM), which includes HSC precursors. Expression of pea3 and $f g f r 4$ was reduced following Wnt16 knockdown, which we previously showed is required for HSC emergence by its regulation of the Notch ligands $d l c$ and $d l d$ in the developing somites $^{28}$. Epistasis experiments demonstrated that ectopic activation of FGF signalling could rescue HSC specification in wnt16 morphants. Within the somite, FGF signalling is therefore required downstream of Wnt16 function for HSC development. Blockade of FGF signalling led to loss of dlc expression, but did not alter dld expression. Loss of HSCs following ablation of FGF signalling was restored by ectopic Notch activation. More specifically, overexpression of dlc mRNA rescued HSC emergence following loss of FGF signalling, demonstrating that FGF function is required for HSC emergence through its regulation of $d l c$ expression. Finally, disappearance of HSCs following knockdown of Fgfr4 indicated that this receptor acts as a specific relay between Wnt16 and Dlc in the somite. Taken together, these results refine our understanding of the signalling cascades necessary within the somite to instruct HSC fate in the neighbouring PLM, and should inform studies seeking the cues necessary to pattern HSCs in vitro from pluripotent precursors.

\section{Results}

FGF signalling is required for HSC specification. To examine a potential role for FGF signalling in HSC development, we used transgenic zebrafish in which FGF signalling can be conditionally abrogated by heat-shock induction of a dominant-negative Fgfr1-EGFP fusion protein $(h s p 70: d n-f g f r 1)^{27}$. To induce dominant-negative Fgfr1, we administered heat-shocks to transgenic animals during several different windows of development. Experimentally perturbed and wild-type (wt) embryos were examined from the outcrossed progeny of heterozygous $h s p 70: d n-f g f r 1$ fish and wt animals, resulting in $50 \%$ transgenic and $50 \%$ wt controls. As FGF signalling is critical for early vertebrate development including mesodermal patterning and somitogenesis ${ }^{29,30}$, early induction of the $d n-f g f r 1$ transgene before $10 \mathrm{~h}$ post fertilization (hpf) led to gross embryonic defects (Supplementary Fig. 1a). However, heat induction during somitogenesis at $12 \mathrm{hpf}$ (5 somites) using optimized heat-shock conditions $\left(38^{\circ} \mathrm{C}, 20 \mathrm{~min}\right)$ led to robust and specific loss of HSCs (Fig. 1a-d; Supplementary Fig. 1c). Whole-mount in situ hybridization (WISH) with the definitive HSC markers runx 1 and $c m y b^{31-33}$ indicated that inhibition of FGF signalling at $12 \mathrm{hpf}$ led to a near complete loss of runx 1 expression at $26 \mathrm{hpf}$ and $c m y b$ at $35 \mathrm{hpf}$, when compared with wt animals (Fig. 1a-d). Interestingly, $>50 \%$ of hsp70:dn-fgfr 1 transgenic embryos induced at $12 \mathrm{hpf}$ showed robust loss of runx1 expression, whereas blockade of FGF signalling at $15 \mathrm{hpf}$ (10 somites) or $17 \mathrm{hpf}$ (15 somites) showed little alteration in runx1 expression (Fig. 1e). Quantitative RT-PCR (qPCR) using $26 \mathrm{hpf}$ wt and $h s p 70: d n-f g f r 1$ transgenic embryos showed that runx1 expression was reduced in the absence of Fgf signalling at $12 \mathrm{hpf}$ (Supplementary Fig. 1b). This blockade of FGF signalling at $12 \mathrm{hpf}$ yielded no alteration in the number of somites during somitogenesis (Supplementary Fig. 2h,i), and runx1 expression in $h s p 70: d n-f g f r 1$ transgenic embryos was similarly reduced at later developmental stages (comparing $30 \mathrm{hpf}$ embryos with $26 \mathrm{hpf}$ embryos; Supplementary Fig. 1d), suggesting that loss of HSCs following FGF blockade was not caused by aberrant developmental rate. We further quantified the number of $\mathrm{cmyb}^{+}$HSCs in the DA (yellow arrowheads in Fig. 1c,d) and observed that FGF signalling inhibition at $12 \mathrm{hpf}$, but not at later time points, perturbed HSC formation, consistent with our runx1 analyses (Fig. 1f). Further quantification of emerging HSCs using triple transgenic cmyb:EGFP; $k d r l: R F P$; $h s p 70: d n-f g f r 1$ embryos demonstrated that $c m y b^{+} k d r l^{+}$double-positive HSCs were significantly reduced after heat induction at $12 \mathrm{hpf}$ compared with wt (Fig. 1g-i). In addition, at 4 and 5 days post fertilization (dpf), expression of $c m y b$ and ragl, markers of developing $\mathrm{T}$ lymphocytes in the thymus, was largely lost following the ablation of FGF signalling at $12 \mathrm{hpf}$ (Fig. $1 \mathrm{j}-\mathrm{m}$ ). Since thymocyte production requires upstream HSCs, these results indicate that FGF signalling is required for HSC development.

To more precisely determine the critical time window that FGF signalling affects HSC formation, we investigated the expression of dominant-negative Fgfr1-EGFP fusion proteins by confocal 

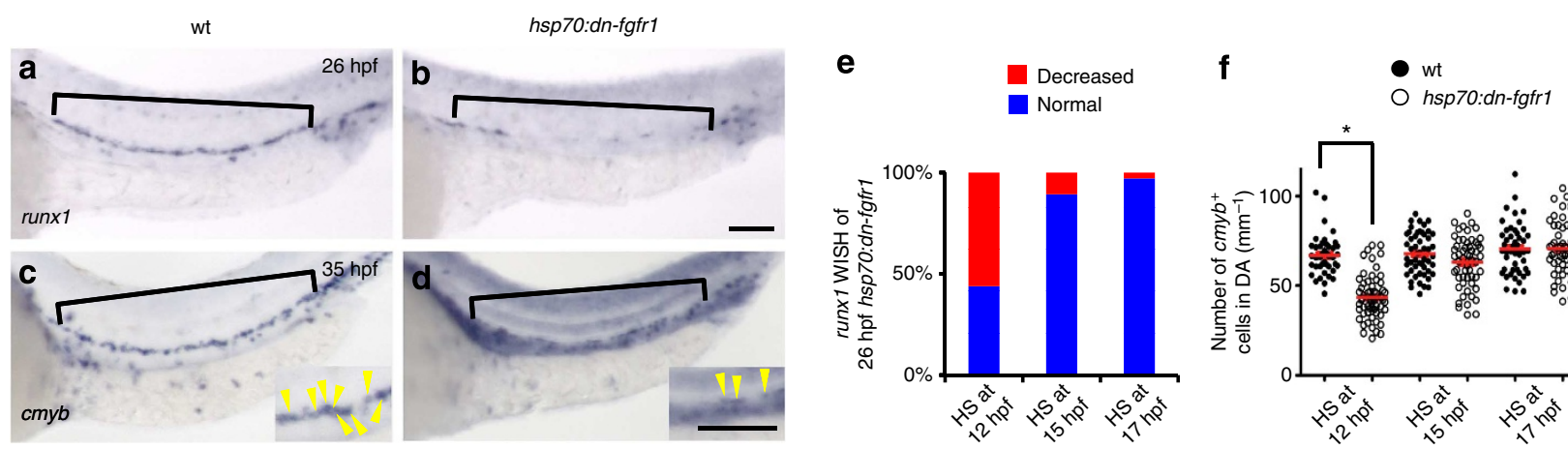

wt
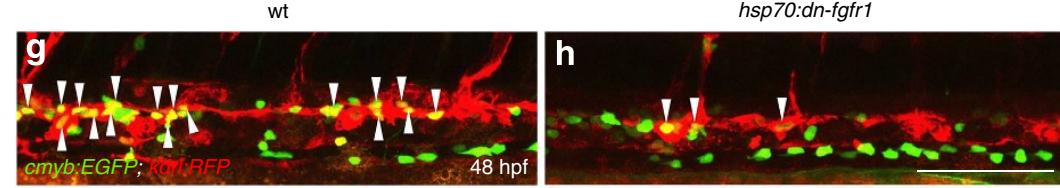

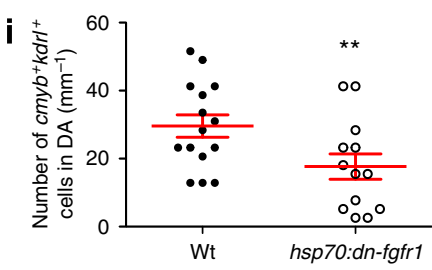

wt
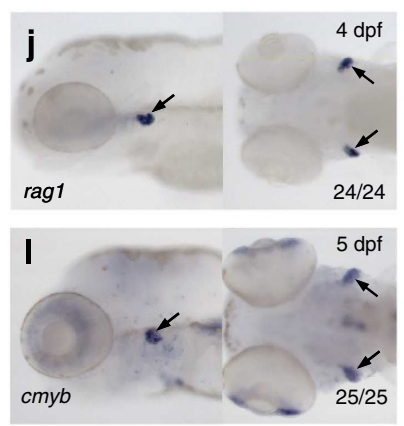

hsp70:dn-fgfr1
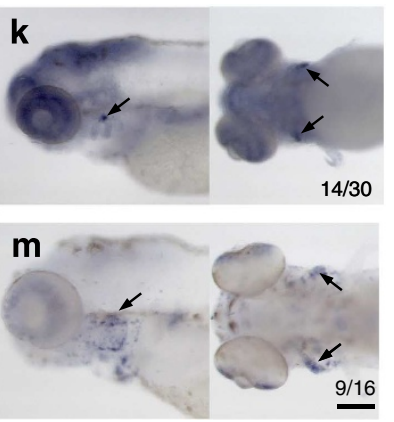
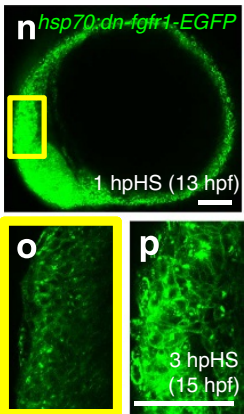
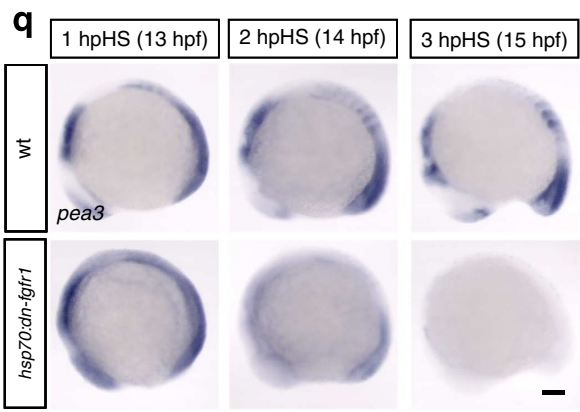

Figure 1 | FGF signalling is required for HSC specification during mid-somitogenesis stages. (a-d) hsp70:dn-fgfr transgenic embryos, heat induced at $12 \mathrm{hpf}\left(38^{\circ} \mathrm{C}, 20 \mathrm{~min}\right.$ ) visualized by WISH for HSC markers runx1 (black brackets) (a,b) and cmyb (black brackets and yellow arrowheads in high magnification images) (c,d) in aortic haemogenic endothelium compared with wt embryos. (e) runx1 phenotype percentages in hsp70:dn-fgfr1 animals following heat-shock at the indicated stages (12 hpf, $n=82 ; 15$ hpf, $n=26 ; 17 \mathrm{hpf}, n=38$ ). (f) Quantification of 35 hpf cmyb ${ }^{+}$cells (yellow arrowheads in c,d) in the DA of wt and hsp70:dn-fgfr1 induced at $12 \mathrm{hpf}$ (wt, $n=42 ;$ hsp70:dn-fgfr1, $n=61$; ${ }^{\star} P$-value $<0.00001$, significantly different from wt, Student's t-test), $15 \mathrm{hpf}$ (wt, $n=52 ; \mathrm{hsp} 70: \mathrm{dn}$-fgfr1, $n=56$ ), and $17 \mathrm{hpf}$ ( wt, $n=48 ; \mathrm{hsp} 70: d n$-fgfr1, $n=54$ ). Red lines indicate mean \pm s.e.m. (g,h) Confocal microscopy images of $48 \mathrm{hpf}$ double-positive HSCs (white arrowheads) in cmyb:EGFP; kdrl;RFP; hsp70:dn-fgfr1 animals following heat-shock at $12 \mathrm{hpf}$. (i) Quantification of $48 \mathrm{hpf} \mathrm{cmyb}^{+} \mathrm{kdrl}{ }^{+}$double-positive HSCs in the DA of wt and hsp70:dn-fgfr1 animals induced at $12 \mathrm{hpf}$ (wt, $n=15 ; \mathrm{hsp} 70: \mathrm{dn}$-fgfr1, $n=13$;

${ }^{\star \star} P$-value $<0.005$, significantly different from wt, Student's $t$-test). (j-m) Lateral and ventral view of WISH images using $h s p 70: d n$-fgfr 1 transgenic embryos heat-induced at $12 \mathrm{hpf}$ for rag1 at $4 \mathrm{dpf}(\mathbf{j}, \mathbf{k})$ and cmyb at $5 \mathrm{dpf}(\mathbf{I}, \mathbf{m})$ in thymus (black arrows) compared with wt embryos. (n-p) Confocal microscopy images of hsp70:dn-fgfr1 embryos heat-shocked at $12 \mathrm{hpf}$. Expression of dominant-negative Fgfr1-EGFP at $13 \mathrm{hpf}(\mathbf{n}, \mathbf{0})$ and $15 \mathrm{hpf}(\mathbf{p})$. (o) The magnified region boxed in yellow shown in (n). (q) WISH for the FGF target pea3 in wt (top row) and hsp70:dn-fgfr embryos (bottom row) at 13, 14, and 15 hpf after heat-shock at $12 \mathrm{hpf}$. Scale bar $=100 \mu \mathrm{m}$.

microscopy. Membrane-localized EGFP was observed within an hour of heat-shock (Fig. 1n,o) and robustly expressed by $3 \mathrm{~h}$ (Fig. 1p). In parallel, we also examined expression of a known target of FGF signalling, pea3 (refs 34,35) (Fig. 1q). Concomitant with the expression of dominant negative Fgfr1-EGFP fusion protein, pea3 expression was severely reduced at $2 \mathrm{~h}$ post heat-shock (hpHS), and absent by $3 \mathrm{hpHS}$. Taken together, these data indicate that the temporal developmental window where FGF signalling is required to specify HSCs is during mid-somitogenesis between 14 and $17 \mathrm{hpf}$.

To determine whether failure of HSC specification in induced $h s p 70: d n-f g f r l$ animals was due to incorrect specification of other mesodermal tissues, we examined expression of the PLM markers scl, fli 1 and $l m o 2$ at $15 \mathrm{hpf}$ (Fig. 2a,b; Supplementary Fig. 2a,b), the ventral mesoderm marker $c d x 4$ (Fig. 2c,d), the notochord marker shh and its targets $p t c 1$ and $p t c 2$ (Fig. 2e,f; Supplementary Fig. $2 \mathrm{c}, \mathrm{d}$ ), the somitic markers desma and vegfa (Fig. 2g,h;
Supplementary Fig. $2 \mathrm{~g}$ ), the primitive erythrocyte marker, gatal (Fig. $2 \mathrm{k}, \mathrm{l}$ ), and a pan-leukocyte marker, l-plastin (Fig. $2 \mathrm{~m}, \mathrm{n}$ ). We observed no gross alterations in any of these tissues or cells. Similarly, convergence of pre-haematopoietic mesoderm was not significantly affected (Fig. 2i,j; Supplementary Fig. 2j). Importantly, expression of the pan-vascular markers $k d r l$, fli1 and $c d h 5$ was normal at $26 \mathrm{hpf}$ (Fig. 2o-r: Supplementary Fig. 2f,k), as was efnb2a, a marker of aortic fate commitment (Fig. 2s,t; Supplementary Fig. 2k), indicating that the observed HSC defects are not likely a result of improper aortic specification.

FGF signalling is active in the somites but not in the PLM. On refining the temporal window in which FGF signalling was required for HSC specification, we investigated what tissues were receiving FGF signals in this time frame to determine whether or not HSC specification might require FGF cell autonomously. 
wt
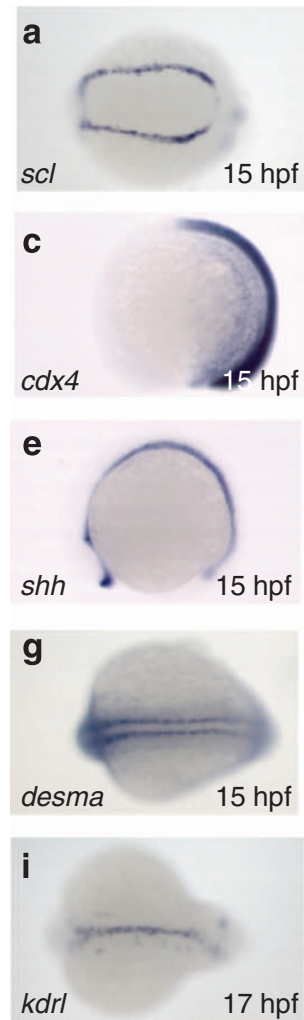

hsp70:dn-fgfr1

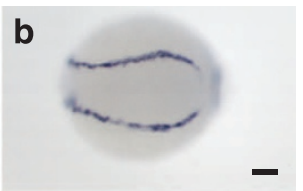

d

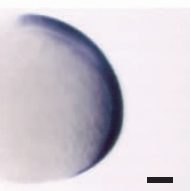

f

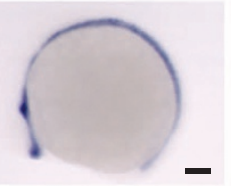

h

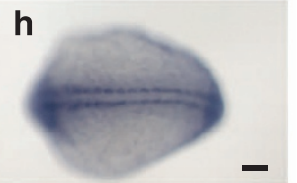

j

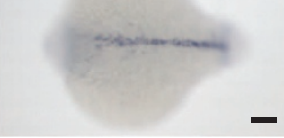

wt
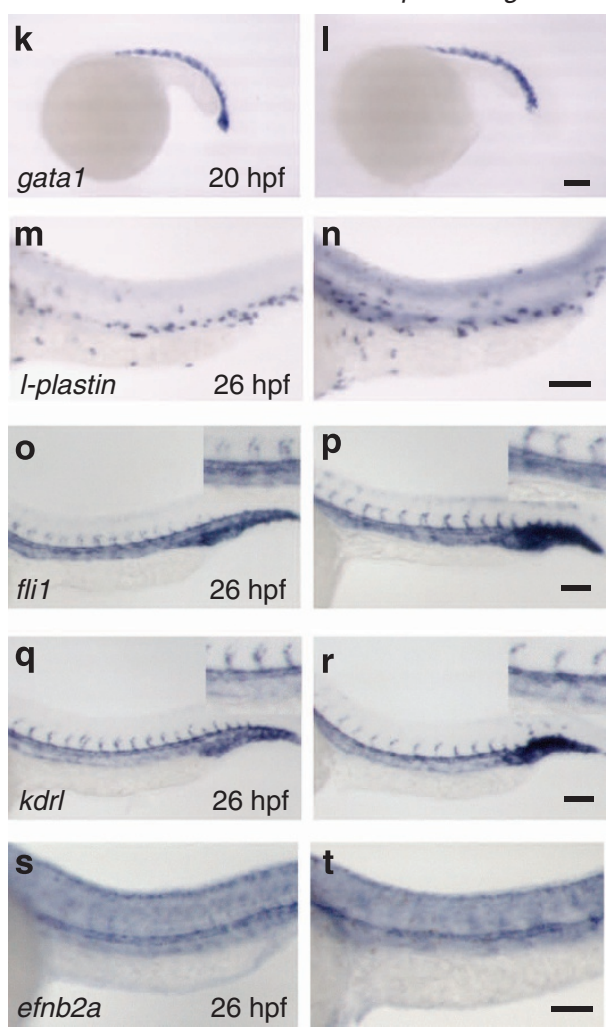

hsp70:dn-fgfr1
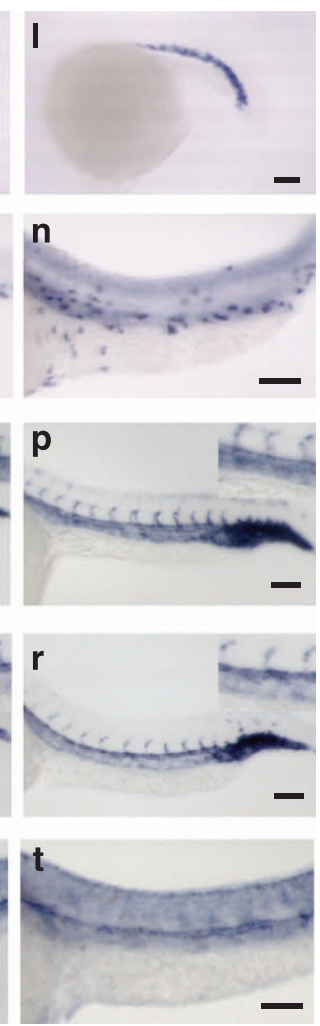

$38^{\circ} \mathrm{C} 20 \mathrm{~min}$ at $12 \mathrm{hpf}$

Figure 2 | The effect of FGF signalling on HSC formation is specific. WISH of wt and induced hsp70:dn-fgfr1 at 12 hpf with the PLM marker scl $(\mathbf{a}, \mathbf{b})$ at $15 \mathrm{hpf}$, the ventral mesoderm marker $c d \times 4$ at $15 \mathrm{hpf}(\mathbf{c}, \mathbf{d})$, the floorpalate and notochord marker shh at $15 \mathrm{hpf}(\mathbf{e}, \mathbf{f})$ and the somite marker desma at $15 \mathrm{hpf}(\mathbf{g}, \mathbf{h})$, the endothelial marker $\mathrm{kdrl}$ at $17 \mathrm{hpf}(\mathbf{i}, \mathbf{j})$, the primitive erythroid marker gatal at 20 hpf $(\mathbf{k}, \mathbf{I})$, the primitive leukocyte marker l-plastin at $26 \mathrm{hpf}(\mathbf{m}, \mathbf{n})$, the endothelial markers flit and $k d r l$ at $26 \mathrm{hpf}$ (o-r; expression in the aorta and vein with higher magnification included), and the arterial marker efnb2a at $26 \mathrm{hpf}(\mathbf{s}, \mathbf{t})$. Scale bar $=100 \mu \mathrm{m}$.

First, we examined expression of the FGF targets pea 3 and erm at $15 \mathrm{hpf}$ and observed that their expression localized mainly to somites (Supplementary Fig. 3). To more precisely determine the expression pattern of FGF target genes, we utilized two-colour enzymatic or fluorescence WISH (FISH) using pea3 and erm along with markers of PLM, which contains the precursors of HSCs ${ }^{5,36-38}$ (Fig. 3a,d-f). Expression of pea3 and erm was restricted primarily to the somites and pre-somitic mesoderm, and did not overlap with $\mathrm{fli}^{+}$or $\mathrm{lmo}^{+}$PLM at $15 \mathrm{hpf}$, indicating that FGF signalling required for HSC specification is active in somites but not in the pre-endothelial mesoderm.

As FGF signalling is transduced by dimerized ligand-bound FGFRs ${ }^{39}$, identifying the spatial distribution of individual receptors provides excellent resolution as to which cells are experiencing FGF signalling in addition to the expression of FGF targets. We examined the expression of all four FGFRs at $15 \mathrm{hpf}$ utilizing WISH and observed no receptor expression in the PLM (Supplementary Fig. 3). In particular, $f g f r 2$ and $f g f r 3$ were not expressed in the embryo posterior, whereas $f g f r 1$ and $f g f r 4$ were expressed in the posterior somites, similar to the FGF targets pea 3 and erm. Two-colour WISH using probes against $f g f r 1$ or $f g f r 4$ and the PLM marker fli1 or $l m o 2$ also demonstrated that neither receptor was expressed in the $\mathrm{fli1}^{+}$or $\mathrm{lmo}^{+}$PLM tissue (Fig. 3b,c,g-l). In addition to whole-animal imaging approaches, we purified PLM precursors $\left(\right.$ fli $\left.^{+}\right)$and somitic cells $\left(\alpha\right.$-actin $\left.{ }^{+}\right)$ from $17 \mathrm{hpf}$ fli1:EGFP or $\alpha$-actin:GFP transgenic embryos by fluorescence-activated cell sorting (FACS) to query expression of $f g f r 1$ and $f g f r 4$ by qPCR. Consistent with our WISH and FISH results, expression of $f g f r 1$ and $f g f r 4$ segregated to the somite fractions (Fig. 3m,n). Taken together, these data suggest that FGF signalling does not act directly on HSC precursors and the requirement for FGF signalling is non-cell autonomous.

Wnt16 acts upstream of FGF signalling in HSC specification. Recent studies from our laboratory showed that Wnt16 regulates the somitic expression of the Notch ligands $d l c$ and $d l d$ whose combined activity is required to specify HSCs during somitogenesis $^{28}$ (Supplementary Fig. 5a). On the basis of spatiotemporal similarities between Wnt16 and FGF activity, we hypothesized the existence of a Wnt16/FGF regulatory network in the somite between 14 and $17 \mathrm{hpf}$. To test this hypothesis, we first examined the expression of wnt16 and the FGF target pea3. As we have seen previously ${ }^{28}$, wnt16 transcript was localized to a relatively anterior-dorsal-lateral compartment of each of the more rostral somites. pea 3 was expressed in the anterior-lateral compartment of more posterior somites, as well as the presomitic mesoderm (PSM) (Fig. 4a-e). As the perdurance and signalling range of Wnt16 protein is unknown, it is difficult to determine whether Wnt16 has a role in somitic maintenance of pea 3 expression that initiates in the PSM. To further analyse potential epistasis between Wnt16 and FGFs, we knocked down wnt16 with an antisense-morpholino (wnt16-MO) and assessed pea3 expression (Fig. 4f,g; Supplementary Fig. 4). Expression of pea3 was robustly downregulated in the formed somites at $17 \mathrm{hpf}$ in the absence of Wnt16, although PSM expression was unaffected. These results indicate that Wnt16 regulates somitic FGF signalling within our noted HSC specification time window. Furthermore, expression 

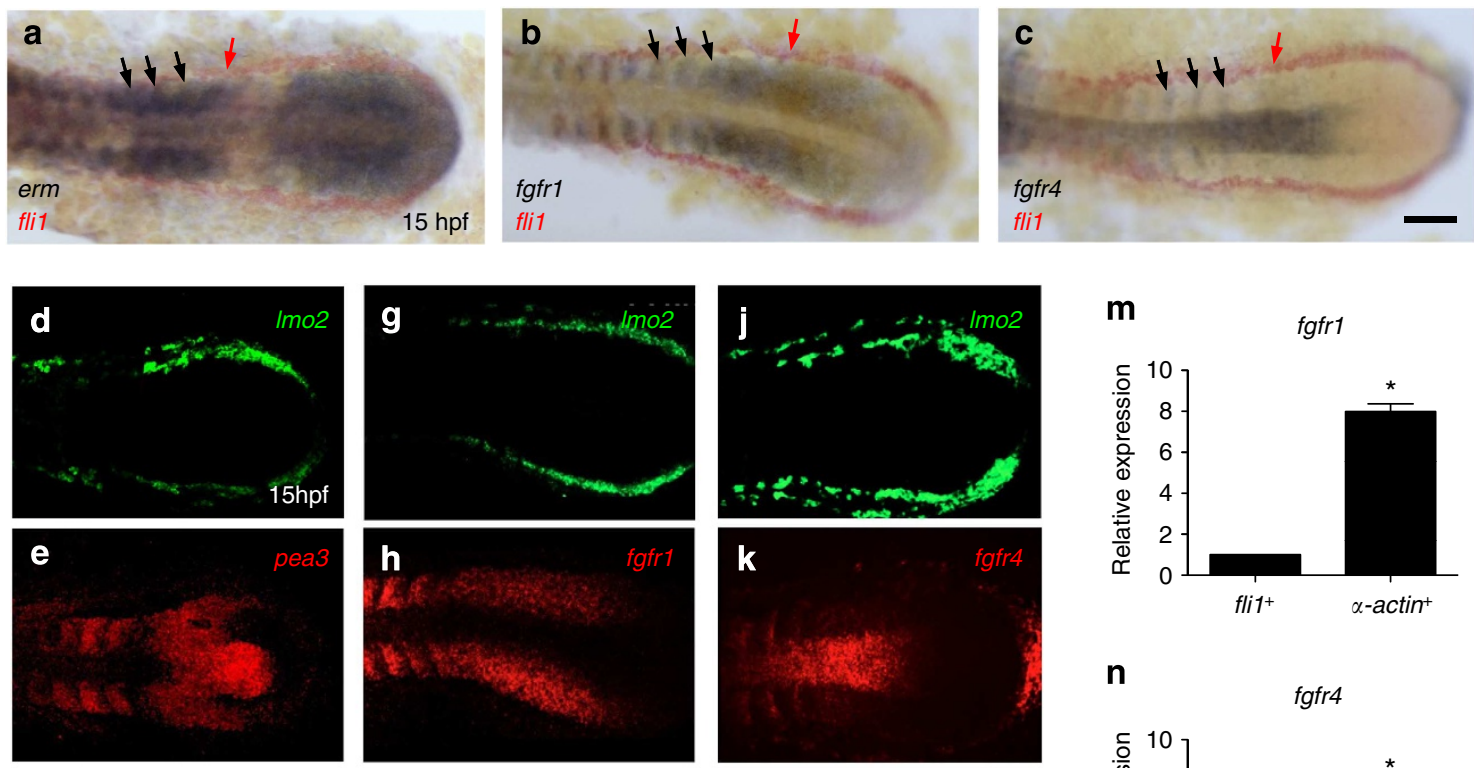

m
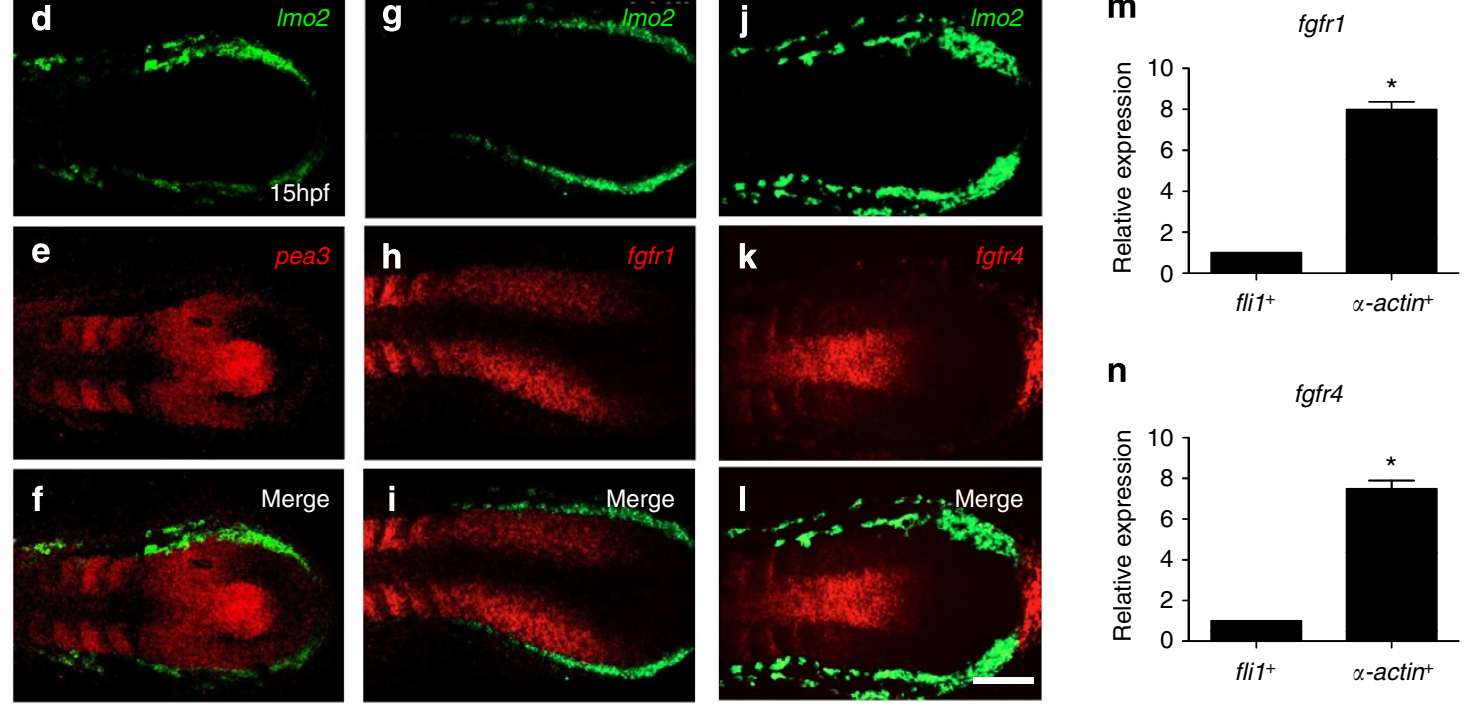

Figure 3 | FGF signalling is active in somitic tissues but not in the pre- endothelial PLM during mid-somitogenesis. (a-c) Images of double enzymatic WISH using probes for the FGF target gene erm (a), fgfr1 (b), and fgfr4 (c) expressed in somites (black arrows) and the PLM marker fli1 (red arrows) at 15 hpf. (d-I) Confocal images of two-colour FISH with the PLM marker Imo2 (green in $\mathbf{d}, \mathbf{g}, \mathbf{j}$ ), fgfrl, fgfr4 and the FGF target gene pea3 (red in $\mathbf{e}, \mathbf{h}, \mathbf{k}$ ) and merged images $(\mathbf{f}, \mathbf{i}, \mathbf{l})$. (m,n) qPCR expression of pre-endothelial PLM flit:EGFP ${ }^{+}$cells and somite-specific $\alpha$-actin:GFP ${ }^{+}$cells at 17 hpf. Expression data were normalized to ef1 $\alpha$ levels $\left(\alpha\right.$-actin ${ }^{+}$fraction, $n=3$; ${ }^{\star} P$ value $<0.005$, significantly different from fli ${ }^{+}$, Student's $t$-test). Scale bar $=100 \mu m$.

of $f g f r 4$ was reduced in wnt16 morphants, while $f g f r 1$ was unchanged relative to control embryos (Fig. 4h,i; Supplementary Figs 4 and 5a). To confirm that Wnt16 acts upstream of FGF signalling to specify HSCs, we utilized a heat-inducible gain-offunction animal carrying a constitutively-active Fgfr1 transgene (hsp70:ca-fgfrl $)^{40}$. Ectopic activation of FGF signalling at $12 \mathrm{hpf}$ recovered normal runx 1 expression in the DA at $26 \mathrm{hpf}$, as well as pea3 expression at $17 \mathrm{hpf}$ in wnt16 morphants (Fig. $4 \mathrm{j}-\mathrm{n}$; Supplementary Fig. 5b). Uninjected but heat-shocked hsp70:cafgfr 1 animals showed no ectopic increase in runx1 expression (Fig. 4k). Similar to the early somitic requirements for Wnt16 and FGF in HSC specification, Vegf signalling is known to function in the development of the vasculature and definitive HSCs during somitogenesis $^{9-11}$. To determine whether Vegf signalling also regulates the activity of FGF signalling, we treated embryos with the Vegf inhibitor ZM306416 during somitogenesis. In contrast to the observed epistasis between Wnt16 and FGF signalling in the somites, pharmacological inhibition of Vegf signalling produced no alteration in expression of either pea3 or fgfr 4 in the somites compared with DMSO-treated controls (Supplementary Fig. 5c, d). Taken together, these results suggest that Wnt16 regulates the FGF signalling pathway during somitogenesis to specify HSCs.

Somitic expression of $d l c$ but not $d l d$ requires FGF signalling. Previous studies suggest that FGF signalling can control Notch ligand expression in the somites through regulation of the transcription factor FoxD5 (ref. 41). Our previous work showed that Wnt16 is required for $d l c$ and $d l d$ expression ${ }^{28}$, but how this regulation is mediated is unknown. Our results above indicate that Wnt16 acts upstream of FGF signalling in HSC specification, suggesting the possibility that FGF signalling might be required for $d l c$ and dld expression. We therefore tested whether FGF signalling might regulate somitic expression of $d l c$ and/or $d l d$. To investigate this question, we blocked FGF signalling with a $12 \mathrm{hpf}$ induction of $h s p 70: d n-f g f r 1$ transgenic animals followed by WISH for $d l c$ and $d l d$ at $15 \mathrm{hpf}$. Loss of FGF signalling led to a loss of somitic $d l c$ expression at $15 \mathrm{hpf}$ (Fig. 5a,b). Likewise, the sclerotome marker foxc1b, which requires somitic $\mathrm{Dlc}^{28}$, was reduced in the absence of FGF signalling (Supplementary Fig. 6a). This effect was specific to the somites, as expression of other Notch ligands and receptors, including $d l c$ in endothelium at $26 \mathrm{hpf}$, was not altered (Supplementary Fig. 6b). In addition, pharmacological inhibition using the FGFR antagonist SU5402 led to a robust reduction of $d l c$ expression when compared with DMSO-treated controls, whereas ectopic activation of FGF signalling enhanced $d l c$ expression in the somites (Fig. $5 c$,d; Supplementary Fig. 6c). In contrast to the effects of $d l c$, somitic expression of dld was not altered following loss of FGF signalling (Fig. 5e-h), indicating that FGF is dispensable for dld expression but required specifically for somitic dlc expression.

Combined expression of $d l c$ and $d l d$ is required for HSC specification downstream of Wnt16, whereas single knockdown of either ligand incompletely blocked HSC formation ${ }^{28}$. We therefore reasoned that the incomplete loss of HSCs following loss of FGF signalling (Fig. 1e) may be due to residual somitic Notch signalling mediated by $d l d$. We therefore tested whether or not combined loss of Dld and FGF function would lead to a more complete loss of HSCs. We analysed the expression of runx1 (26 hpf) and $c m y b$ (32 hpf) in dld-MO injected $h s p 70: d n-f g f r 1$ and wt control embryos heat-shocked at $12 \mathrm{hpf}$ (Fig. 5i-p). Whereas injection of individual dld-MO into wt embryos led to a loss of 

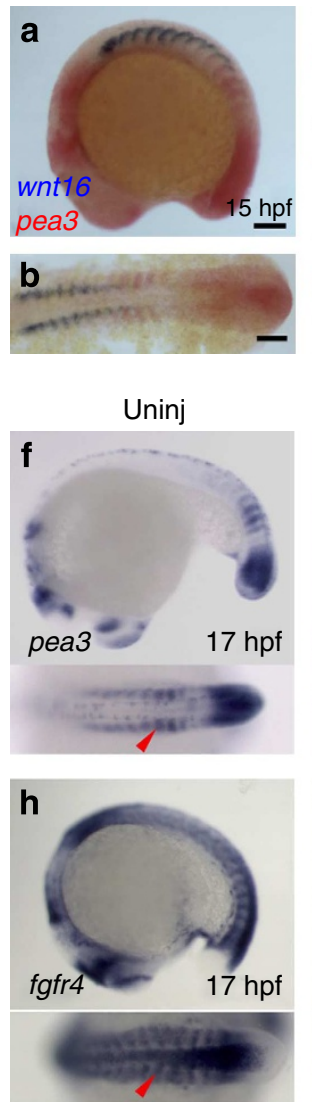
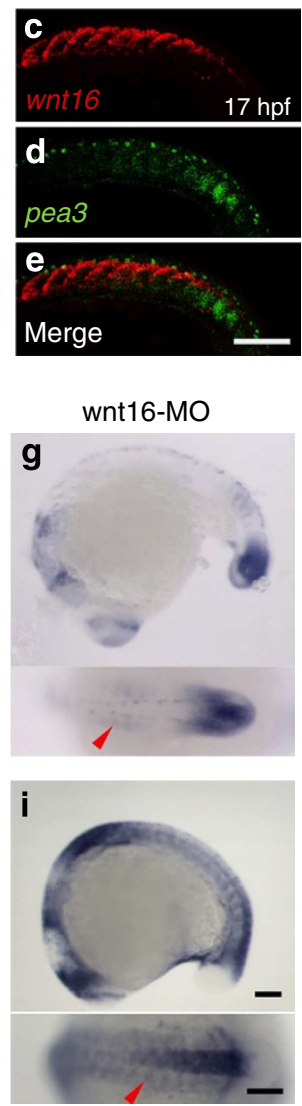
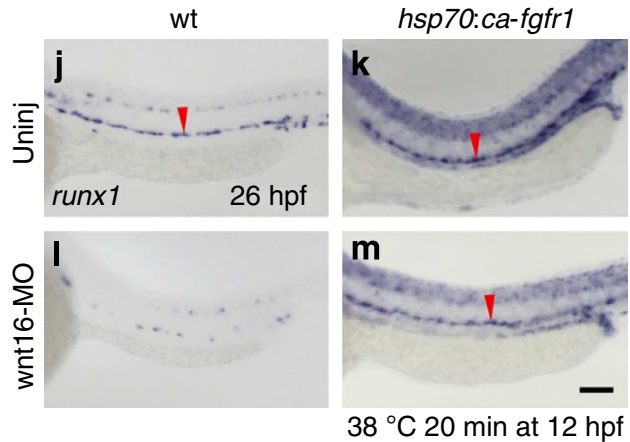

n

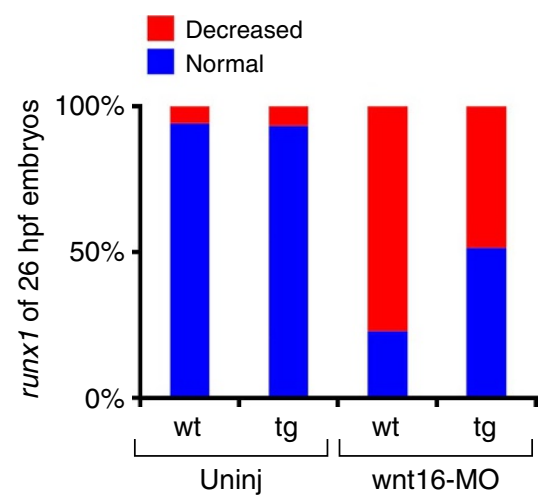

Figure 4 | Wnt16 controls FGF signalling activity. (a,b) Expression of wnt16 (blue) and pea3 (red) at 15 hpf by two-colour enzymatic whole-mount and flat-mount in situ hybridization. (c-e) Confocal images of FISH for wnt16 (red in $\mathbf{c}$ ), pea3 (green in d), or merge (e) at $17 \mathrm{hpf}$. (f-i) Lateral and dorsal views of expression of pea3 $(\mathbf{f}, \mathbf{g})$ and fgfr $4(\mathbf{h}, \mathbf{i})$ in wnt16 morphants at 17 hpf compared with uninjected controls as indicated at top. $(\mathbf{j}-\mathbf{m})$ WISH for runx 1 expression at $26 \mathrm{hpf}$ following ectopic activation of FGF signalling using hsp70:ca-fgfr 1 induced at $12 \mathrm{hpf}\left(38^{\circ} \mathrm{C} 20 \mathrm{~min}\right)$ in wnt $16 \mathrm{morphants}$. (n) runx1 phenotype percentages in wnt16-MO injected hsp70:ca-fgfr1 transgenic embryos (tg) heat-shocked at 12 hpf, compared with wnt16-MO injected wt embryos (uninjected wt, $n=34$; uninjected tg, $n=44$; wnt16-MO injected wt, $n=48$; wnt16-MO injected tg, $n=37$ ). Red arrowheads indicate WISH signals of pea3 and fgfr 4 in the somites $(\mathbf{f}-\mathbf{i})$ and runx 1 in the DA (j-m). Scale bar $=100 \mu \mathrm{m}$.

runx1 and cmyb expression at levels comparable to heat-induced hsp70:dn-fgfrl alone, combinatorial ablation of FGF signalling and Dld function led to significantly lower numbers of HSCs (Fig. 5q,r).

Finally, to confirm that FGF signalling acts upstream of $d l c$ to specify HSCs, we performed rescue experiments in which $h s p 70: d n-f g f r 1$ animals were injected with $d l c$ mRNA at the 1-2cell stage of development followed by heat-shock at $12 \mathrm{hpf}$. As assessed by runx1 and $c m y b$ expression, HSC numbers were significantly rescued following injection of $d l c$ in the absence of FGF signalling (Fig. 6a-f; Supplementary Fig. 7a). To examine this in another way, we performed rescue experiments with transgenic animals whereby Notch signalling can be experimentally activated by heat induction (hsp70:gal4; UAS:NICD$m y c)^{14,42}$. To obtain triple transgenic animals, $h s p 70: d n-f g f r 1$ animals were crossed with hsp70:gal4; UAS:NICD-myc fish. Both dn-Fgfr1 and NICD-myc, transgenes were induced by heat-shock at $12 \mathrm{hpf}$, and $\mathrm{NICD}^{+}$transgenic fish were identified by myc-antibody staining after runxl WISH was performed (Supplementary Fig. 7c). NICD overexpression in $h s p 70: d n-f g f r 1$ embryos led to a mild recovery of runx1 expression (Supplementary Fig. 7b,d). As heat-shock at later time points (15 hpf) showed no loss of HSCs in $h s p 70: d n-f g f r 1$ embryos (Fig. 1e,f), we performed a second heat-shock in triple transgenic embryos to maximize the effects of NICD on HSC specification. A first heat-shock was administered at $12 \mathrm{hpf}$ to ablate FGF signalling by $15 \mathrm{hpf}$. Next, a second heat-induction was performed at $14 \mathrm{hpf}$, as this time point was previously utilized to optimally rescue HSC specification in wnt16 morphants via NICD induction ${ }^{28}$. WISH for runx 1 at $26 \mathrm{hpf}$ demonstrated that activation of Notch signalling almost fully recovered runx $1^{+}$ HSCs following ablation of FGF signalling (Fig. 6g-m). Taken together, these results demonstrate that FGF signalling is required for dlc but not dld expression in somites to regulate HSC specification during somitogenesis.

Fgfr4 acts to relay Wnt16 signals to Dlc to specify HSCs. During early to mid-somitogenesis, knockdown of Wnt16 led to diminished expression of $f g f r 4$ but no alteration of $f g f r l$ in somites (Fig. 4h,i; Supplementary Fig. 5a). Moreover, two-colour FISH demonstrated that $d l c$ was expressed in $f g f r 4^{+}$but not in $f g f r 1^{+}$ somitic tissues (Fig. 7a-f). On the basis of these results, we hypothesized that Fgfr4 may mediate the Wnt16/Dlc signalling cascade to regulate HSC specification. To test this hypothesis, we knocked down the function of $f g f r 4$ using both splicing-blocking (fgfr4-MO1) and translation-blocking (fgfr4-MO2) receptorspecific MOs. We observed that $d l c$ expression in somites at $15 \mathrm{hpf}$ as well as foxc $1 b$ expression in the sclerotome was reduced in both $f g f r 4$ morphants when compared with uninjected and control MO-injected embryos (Fig. 7g-i; Supplementary Figs 4 and $8 \mathrm{~b})$. By contrast, knockdown of $f g f r 1$ led to no alteration in $d l c$ expression (Supplementary Fig. 8a). Finally, to determine whether regulation of $d l c$ via Fgfr4 is upstream of HSC 

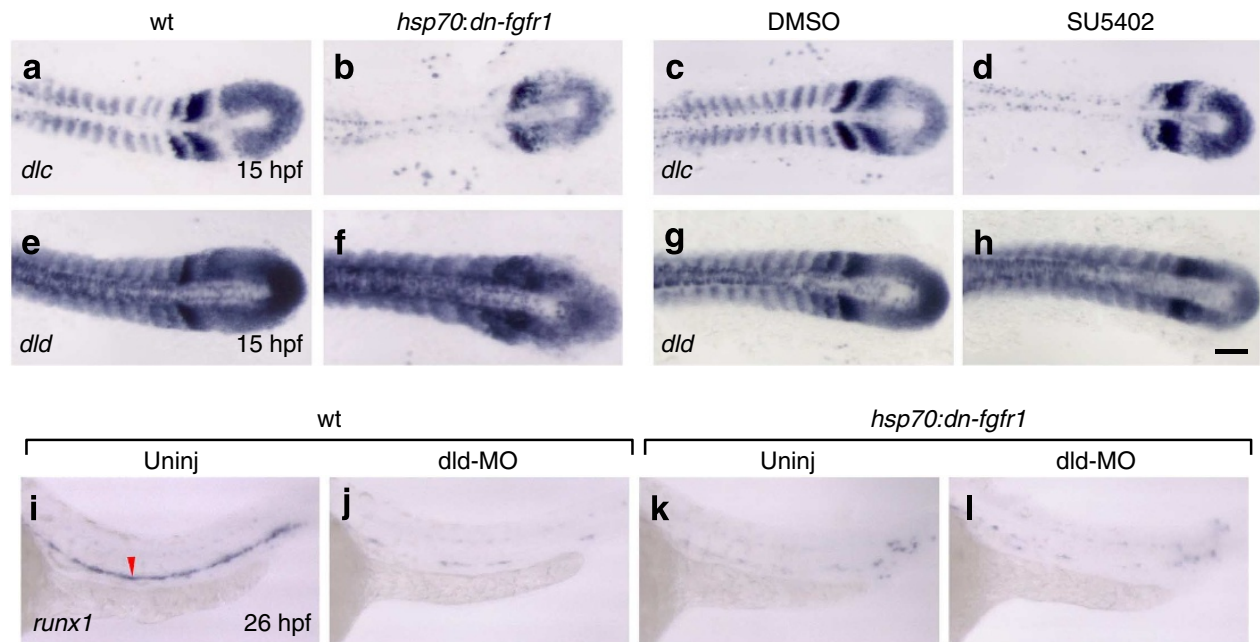

wt
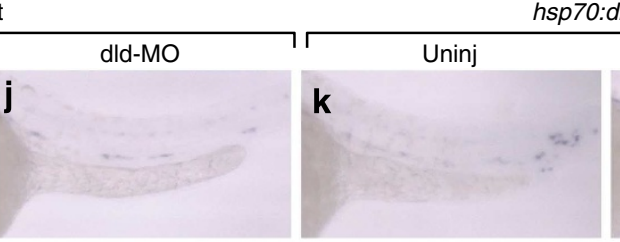

p70:dn-fgfr1
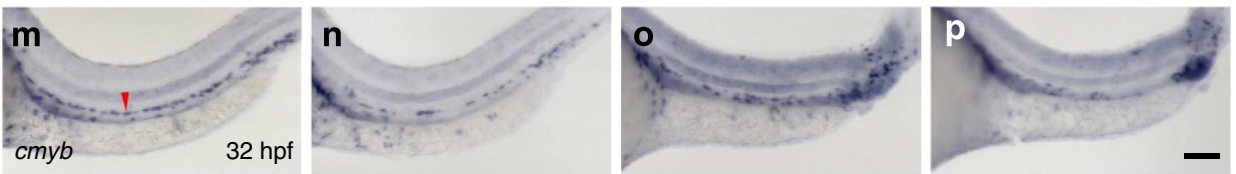

$38^{\circ} \mathrm{C} 20 \mathrm{~min}$ at $12 \mathrm{hpf}$
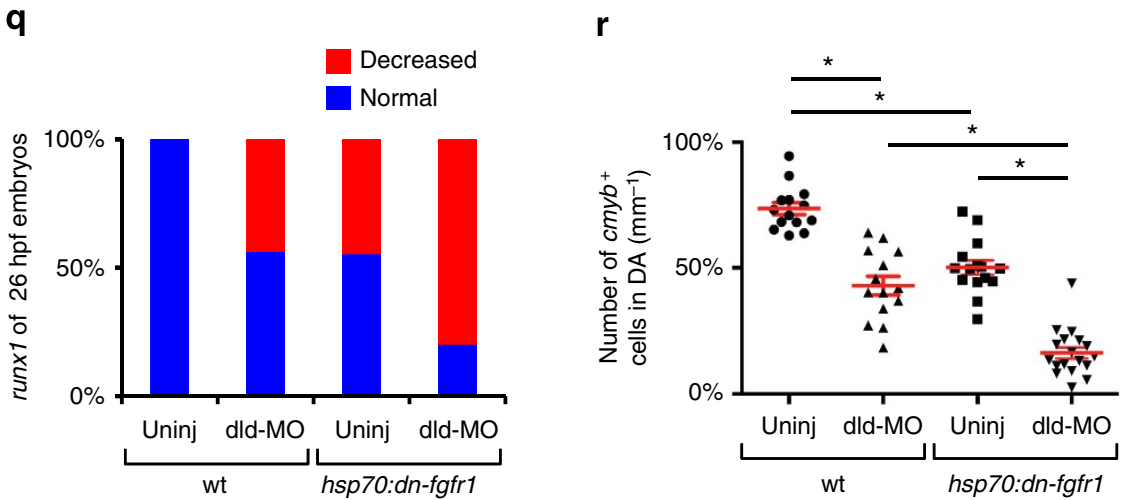

Figure 5 | Somitic dlc expression, but not dld, is regulated by FGF signalling. (a-h) Expression of Notch ligands dlc and dld in 15 hpf $h s p 70: d n-f g f r 1$ animals heat-shocked at $12 \mathrm{hpf}$ compared with wt controls (a,b,e,f) and wt embryos incubated with the FGFR-antagonist SU5402 (12-15 hpf) compared with DMSO-treated controls (c,d,g,h). (i-p) Expression of HSC markers runx1 at $26 \mathrm{hpf}(\mathbf{i}-\mathbf{I})$ and cmyb at $32 \mathrm{hpf}(\mathbf{m}-\mathbf{p})$ in uninjected wt (i,m), dld-MO injected wt (j,n), uninjected hsp70:dn-fgfr1 (k,o) and dld-MO injected hsp70:dn-fgfr1 (I,p) embryos heat-shocked at 12 hpf. Red arrowheads indicate runx1 or cmyb WISH signals in uninjected controls (i,m). (q,r) Percentage of embryos displaying a runx 1 phenotype at $26 \mathrm{hpf}$ ( $\mathbf{q}$; uninjected wt, $n=54 ;$ dld-MO injected wt, $n=41$; uninjected hsp70:dn-fgfrl, $n=58$; dld-MO injected hsp70:dn-fgfr1, $n=45$ ) and quantification of cmyb ${ }^{+}$cells in DA (r; uninjected wt, $n=14$; dld-MO injected wt, $n=14$; uninjected $h s p 70: d n$-fgfr1, $n=15$; dld-MO injected hsp70:dn-fgfr1, $n=18$; mean \pm s.e.m.; ${ }^{\star} P<0.00001$, Student's $t$-test) after blocking dld expression and FGF signalling. Scale bar $=100 \mu \mathrm{m}$.

generation, we analysed HSC marker expression in $f g f r 4$ morphants. Loss of Fgfr4 function led to a marked decrease in the number of runx $^{+}$and $c m y b^{+}$cells along the DA (Fig. 7j-o; Supplementary Fig. 8b,c). Although $f g f r 4$ morphant animals showed slight patterning defects potentially caused by the ablation of $f g f r 4$ at earlier developmental stages ${ }^{43}$, both endothelium and DA appeared normal in the morphants (Fig. 7p-u; Supplementary Fig. 8b). In addition, knock down of $f g f r 4$ using fgfr4-MO2 led to no alterations in shh and vegf expression, whereas expression of the sclerotomal marker foxc $1 b$ was severely reduced (Supplementary Fig. 8d), consistent with our findings in $h s p 70: d n-f g f r 1$ transgenic animals (Fig. 2e,f; Supplementary Fig. $2 \mathrm{~g}$ and $6 \mathrm{a}$ ). Further analysis of HSC emergence using cmyb:EGFP; kdrl:RFP transgenic animals ${ }^{5}$ showed a marked reduction in the number of $c m y b^{+} k d r l^{+}$double-positive HSCs in $f g f r 4$ morphants compared with controls (Fig. $7 \mathrm{v}-\mathrm{y}$ ). Taken together, these results indicate that Fgfr4 plays a pivotal role in mediating the Wnt16/Dlc signalling cascade required for instruction of HSC fate.

\section{Discussion}

Although roles for FGF signalling in primitive haematopoiesis have been described ${ }^{21-23}$, its potential function in the embryonic development of HSCs has not been addressed. Here, we demonstrate that FGF signalling is required for HSC specification through its actions in neighbouring somitic tissues. Our studies suggest that FGF signalling in the somite serves to bridge a signalling cascade between a non-canonical Wnt ligand, Wnt16, and the Notch pathway through its regulation of Dlc (Fig. 8).

Inhibition of FGF signalling using inducible transgenic $h s p 70: d n-f g f r 1$ animals reveals that induction of dn-Fgfr1 at $12 \mathrm{hpf}$ led to significantly fewer specified HSCs. Our strategy for 

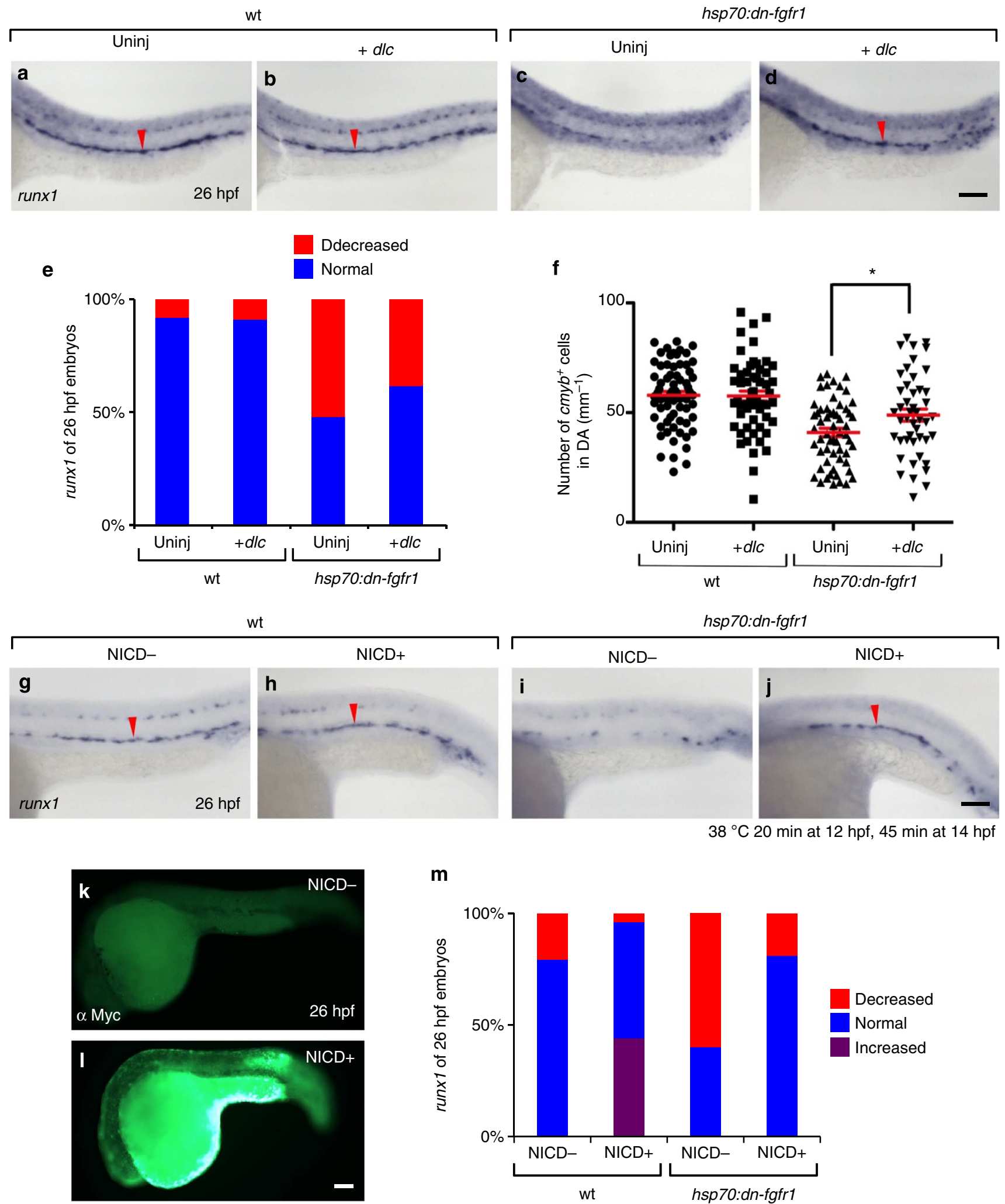

Figure 6 | FGF regulation of HSC specification requires the Notch ligand Dlc. (a-d) Expression of runx 1 in wt and hsp70:dn-fgfr1 embryos injected with $100 \mathrm{pg}$ of dlc mRNA and uninjected controls. Embryos were heat induced at $12 \mathrm{hpf}$. (e,f) Quantitation of defective embryos showing decreased expression of runx1 at $26 \mathrm{hpf}$ (e; uninjected wt, $n=61 ; d l c+w t, n=44$; uninjected hsp70:dn-fgfr1, $n=46 ; d l c+h s p 70: d n-f g f r 1, n=52$ ) and cmyb ${ }^{+}$cells at $32 \mathrm{hpf}$ (f; uninjected wt, $n=68$; dlc + wt, $n=56$; uninjected $h s p 70: d n-f g f r 1, n=58 ; d l c+h s p 70: d n-f g f r 1, n=46$ ) using hsp70:dn-fgfr1 injected with dlc mRNA and followed by heat-shock at $12 \mathrm{hpf}$ (mean \pm s.e.m.; ${ }^{\star} P<0.05$, Student's $t$-test). (g-j) runx1 expression at $26 \mathrm{hpf}$ in hsp70:gal4; UAS:NICD-myc; hsp70:dn-fgfr1 heat-shocked at both 12 and $14 \mathrm{hpf}$. (k,I) NICD + embryos were identified by immunostaining for the myc epitope tag, following WISH. (m) Percentage of embryos with a runx 1 phenotype at $26 \mathrm{hpf}$ in wt or FGF-blocked, with or without enforced NICD expression (NICD- wt, $n=48$; NICD + wt, $n=25$; NICD- hsp70:dn-fgfr1, $n=20 ; \mathrm{NICD}+$ hsp70:dn-fgfr1, $n=21$ ). Red arrowheads indicate runx1 expression in the DA. Scale bar $=100 \mu \mathrm{m}$. 

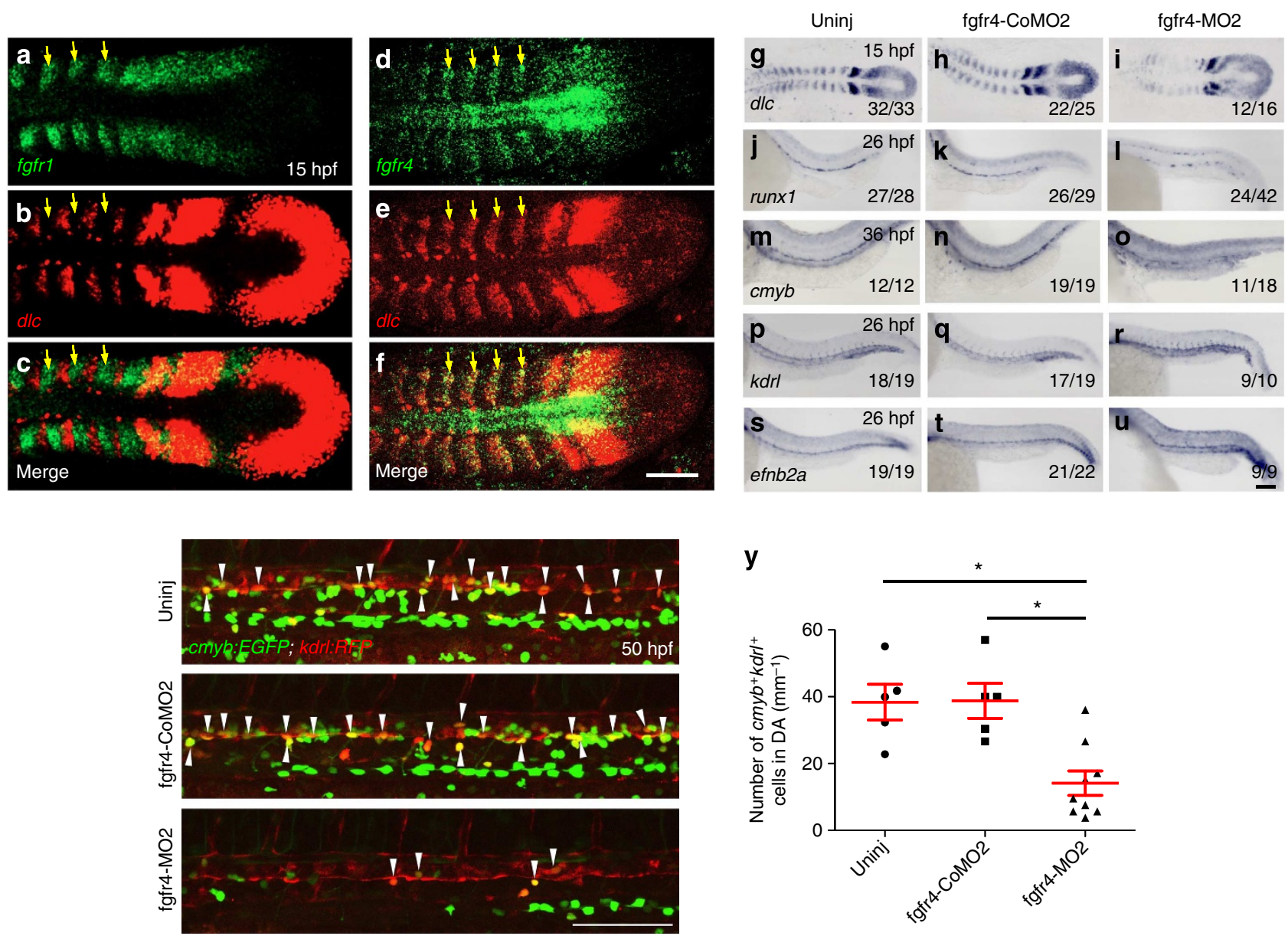

Figure 7 | Fgfr4 is required for somitic dlc expression and HSC specification. (a-f) Single-plane confocal images of two-colour FISH for fgfr1 (a), dlc (b) and merged comparison (c), and fgfr4 (d), dlc (e) and their merged comparison (f) at 15 hpf. Yellow arrows indicate expressions of fgfr1 (a) or fgfr 4 (d) in the somites. (g-u) Expression of somitic dlc at $15 \mathrm{hpf}$. (g-i), runx1 at $26 \mathrm{hpf}(\mathbf{j}-\mathbf{I}), \mathrm{cmyb}$ at $36 \mathrm{hpf}(\mathbf{m}-\mathbf{o})$, the endothelium marker $\mathrm{kdrl}(\mathbf{p}-\mathbf{r})$ and the aortic marker efnb2a (s-u) at $26 \mathrm{hpf}$ in fgfr4-MO2 injected embryos, compared with uninjected and mismatched control MO (fgfr4-CoMO2)- injected siblings. (v-x) Double-positive HSCs (white arrowheads) in kdrl:RFP; cmyb:EGFP transgenics in the fgfr4 morphants (x) compared with uninjected (v) and mismatched control MO injected (w) controls. (y) Quantitation of $50 \mathrm{hpf} \mathrm{cmyb}^{+} \mathrm{kdrl}{ }^{+}$double-positive HSCs in the DA of fgfr4 morphants (v-x) (uninj, $n=5$; control MO, $n=5$; fgfr 4 morphants, $n=9$; ${ }^{\star} P<0.01$, significantly different from uninjected and control MO-injected ones, Student's $t$-test). Scale bar $=100 \mu \mathrm{m}$.

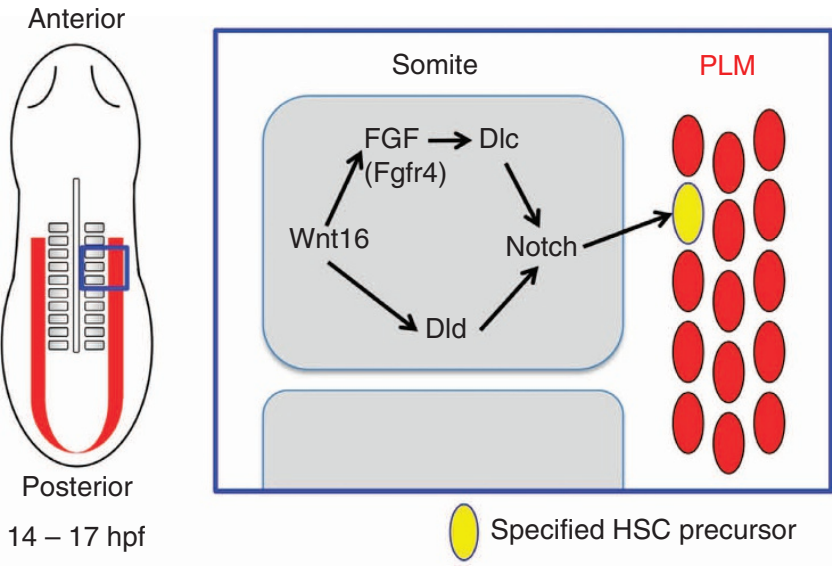

Figure 8 | Model of the early FGF requirement in HSC specification. Cartoon depicts a dorsal view during mid-somitogenesis (14-17 hpf). FGF signalling specifies HSCs by relaying signals between Wnt16 and Dlc in the somite. Fgfr4 is the key receptor that mediates this Wnt16/Notch HSC specification pathway. time-specific FGF inhibition allowed us to bypass earlier known requirements for FGF signalling in mesoderm patterning and primitive haematopoiesis ${ }^{23,30,44,45}$, and directly examine the later time points proximal to HSC specification. These results define a critical requirement for FGF signalling in HSC specification from 14 to $17 \mathrm{hpf}$ during somitogenesis, after its role in mesoderm formation and primitive blood cell development has passed. Interestingly, as presented in the companion paper ${ }^{46}$, even later modulation of the FGF pathway at $20.5 \mathrm{hpf}$ shows an opposite role for FGF signalling, limiting emergence of HSCs from the aortic floor. Our studies indicate that FGF signalling during somitogenesis mediates Wnt16 activation of Dlc within the somite, while our collaborators demonstrate that FGFs negatively regulate HSC formation by restricting a necessary prohaematopoietic BMP signal at $20.5 \mathrm{hpf}$. Together with past work, these results demonstrate that the regulation of HSC formation by FGF signalling is dynamic, with multiple independent roles present in distinct temporal windows.

Sonic Hedgehog (Shh) and Vegf play key roles in patterning the DA and regulating emergence of HSCs. During early zebrafish somitogenesis, Shh activates expression of Vegf that in turn is required for the expression of Notch1 in the arterial 
vasculature $^{31,47,48}$. Notch1 is required cell autonomously in the haemogenic endothelium for HSC specification ${ }^{15}$. Our current studies indicate that Fgf signalling does not interact with this pathway. Expression of $s h h$, as well as its targets $p t c 1$ and $p t c 2$, was not altered following ablation of FGF signalling. No alteration of vegfa was observed in somites of FGF-inhibited embryos, and, conversely, the FGF target pea3 was normal in embryos treated with Vegf inhibitors. Moreover, not only did the vasculature develop morphologically normally, endothelial expression of the Notch ligands $d l c$ and dll4, as well as the receptors notch $1 b$ and notch3, was normal in the absence of FGF signalling. Our results thus indicate that the requirement for FGF signalling in HSC specification is independent of the Shh and Vegf pathways, but rather operates temporally in parallel.

A growing body of evidence supports the idea that gene expression and patterning within the somites play a critical role in HSC specification, but many details remain mysterious. We previously identified an important role for the non-canonical Wnt ligand, Wnt16 in HSC specification. Loss of Wnt16 led to loss of the two key Notch ligands $d l c$ and $d l d$ in the somite. These in turn regulate HSC specification, but whether or not each acts directly on the haemogenic endothelium remains to be determined.

Multiple lines of evidence supported the idea that the temporal 14-17 hpf requirement observed for FGF signalling in HSC specification is operational within the formed somites. The FGF targets pea 3 and erm are expressed in the somites, but not the PLM; so are the key receptors, $f g f r 1$ and $f g f r 4$. As the FGF signalling requirement we have defined here does not interact with the Shh/Vegf/Notch pathway, we examined the possibility that it interacts with the Wnt16/Dlc/Dld pathway ${ }^{28}$, which is also operational within the somites. Knockdown of Wnt16 reduces the expression of pea 3 and $f g f r 4$, and this loss of expression could be rescued by enforced activation of FGF signalling, revealing Wnt16 is required to turn on or potentiate somitic FGF signalling. These results reveal a novel non-canonical Wnt/Fgf signalling axis upstream of HSC specification.

To better locate where FGF signalling acts in the Wnt16/Dlc/ Dld/HSC specification pathway, we examined the ability of Fgf to regulate expression of the pivotal somitic Notch ligands $d l c$ and dld. This possibility seemed plausible, given previous studies that have suggested that somitic Notch ligand expression is regulated by FGF signalling ${ }^{41}$. Interestingly $d l c$, but not $d l d$, requires FGF signalling downstream of Wnt16. We have determined that the key FGFR in this pathway is Fgfr4. Although both $f g f r 1$ and $f g f r 4$ are expressed in the somites, only $f g f r 4$ expression directly overlaps that of $d l c$, and only knockdown of Fgfr4 has significant effects on $d l c$ expression. Our results strongly suggest the possibility that Fgfr4 cell autonomously regulates expression of Dlc, but we cannot exclude the possibility of paracrine relay signalling within the Fgfr4 ${ }^{+}$population. Consistent with the notion that Fgfr4 is active within the somites and not in the endothelium, knockdown of Fgfr4 yields changes in somitic but not vascular gene expression, leaving endothelial and arterial markers intact.

Importantly, our results locate a requirement for FGF signalling within a now better-defined somitic Wnt16/FGF/Notch HSC specification pathway. FGF signalling here works to activate expression of $d l c$ but not $d l d$, which represents a parallel input downstream of Wnt16, but not evidently regulated through FGF. runx $1^{+}$HSCs are partially lost in FGF-inhibited conditions and this loss can be rescued by injection of $d l c$ mRNA. Consistent with the bifurcation of the pathways downstream of Wnt16 at the point of FGF signalling, HSCs are partially ablated in the absence of FGF signalling, and fully ablated when $d l d$ is knocked down in parallel to FGF inhibition.
The identification of Fgfr4 as a receptor specifically required for HSC generation begs the question of which FGF ligands are active in this process. Our results demonstrate that Wnt16 is critical for cellular reception of the FGF signal, by activating expression of $f g f r 4$, but do not exclude the possibility that it also activates expression of key ligands and downstream signals transduction factors. The FGF ligands FGF1, FGF8 and FGF17 are expressed in developing somites ${ }^{49-51}$. FGF1 increases the generation of long-term repopulating HSCs in vitro ${ }^{25}$ and is required for primitive haematopoiesis in zebrafish embryos ${ }^{49}$. FGF8 has a critical role in mesoderm formation and patterning ${ }^{52,53}$ and zebrafish studies with $f g f 8$ mutant animals (ace) indicate that FGF8 is required for somitogenesis ${ }^{50}$. Likewise, FGF17 potentially plays a role in somite patterning ${ }^{51}$. However, because each of these ligands also has earlier developmental functions, published loss-of-function studies were limited in determining the role of FGFs during the later developmental stages important for haematopoietic specification. Therefore, inducible genetic tools are necessary to further analyse individual roles of FGF ligands in HSC specification.

Although our studies have precisely defined a specific temporal window of somitic signalling required for HSC specification, the relay factors that signal to the adjacent PLM to specify HSC precursors remain to be defined. Multiple studies have now defined specific genetic perturbations in which both sclerotome and HSC specification are abrogated, suggesting the possibility that HSC specification somehow requires the sclerotome. The sclerotome is the ventromedial compartment of developing somites, which gives rise predominantly to elements of the axial skeleton. Sclerotome has been postulated to also give rise to the vascular smooth muscle cells (VSMCs) that sheath the dorsal aorta $^{54-57}$, but others have found a different origin ${ }^{58}$. Similar to wnt16 morphants, and loss of function in either or both of the Notch ligands Dlc and Dld ${ }^{28}$, loss of FGF signalling by either global ablation or specific knockdown of Fgfr4 led to loss of sclerotomal tissues, suggesting that the Wnt16-FGF-Notch signalling cascade in the somite directs sclerotome development that may subsequently relay necessary signals from the somites to the PLM. Our recent results suggest that one such relay signal is another iteration of Notch signalling, where Notch ligands presented by the sclerotome make direct contact with the shared vascular precursors of HSCs as they migrate to form the pre-aortic vascular cord ${ }^{59}$. In addition, subsequent signals may be carried by VSMC precursors derived from the sclerotome ${ }^{1}$. To further address the role of the sclerotome, more tissue-specific transgenic reagents will be necessary to investigate its function and behaviour during HSC development.

A second population of somite-derived cells has recently been described that has been suggested to be required for HSC specification, the 'endotome', a pax3 ${ }^{+}$population near the septa (vertical somite boundaries), which incorporates into the maturing endothelium ${ }^{60}$. A similar population has previously been described in chick ${ }^{61}$ and possibly mouse ${ }^{55}$, but was not previously implicated in HSC specification. The Wnt16/FGF/ Notch pathway appears to be discrete from the endotomal pathway, most notably in that the affected regions of the somite are different $\left(\right.$ foxc $1 b^{+}$sclerotome versus pax3 $3^{+}$septa), but we cannot rule out the possibility that Wnt16/FGF/Notch regulates both endotomal and sclerotomal contributions to HSC specification. The fact that in choker mutants, which display an increased contribution of somite-derived cells to the endothelium of the dorsal aorta, wnt16 expression is nearly ablated suggests that the choker mutation (meox1a) short circuits the requirement for Wnt16 (ref. 60), for example by leading to an upregulation of FGF signalling or enhanced presentation of Notch ligands. 
In conclusion, our studies suggest that FGF signalling through Fgfr4 is required to mediate a signalling cascade between Wnt16 and Dlc in the developing somite to specify HSCs. These findings provide a better understanding of HSC ontogeny and have elucidated a novel set of regulatory inputs necessary for HSC development. Importantly, our findings shed light on pathways that can be potentially modulated to instruct in vitro production of HSCs from pluripotent precursors, a process that has proven to be extremely challenging.

\section{Methods}

Zebrafish husbandry. Wild-type $\mathrm{AB}^{\star}$ and transgenic $h s p 70: d n-f g f r 1$ $\left(T g(h s p 70 l: d n f g f r 1-E G F P)^{p d 1}\right)^{27},\left(T g(c m y b: E G F P)^{z f 169}\right)^{62},\left(T g(k d r l: R F P)^{l a 4}\right)^{63}$, hsp70:ca-fgfr1 (Tg(hsp70l:Xla.fgfr1, cryaa:DsRed $\left.)^{p d 3}\right)^{40}$, UAS:NICD-myc $\left(T g(5 x U A E-E 1 b: 6 x M Y C-n o t c h 1 a)^{k c a 3}\right)^{42}$, hsp70:gal4 $\left(T g(-1.5 h s p 70 l: G a l 4)^{k c a 4}\right)^{42}$, fli1:EGFP (Tg(fli1a:EGFP) $\left.)^{y 1}\right)^{64}, \alpha$-actin:GFP (Tg(actc1b:GFP $\left.)^{z f 13}\right)^{65}$ zebrafish embryos and adult fish were raised in a circulating aquarium system (Aquaneering) at $28^{\circ} \mathrm{C}$ and maintained in accordance with UCSD Institutional Animal Care and Use Committee (IACUC) guidelines. For heat-shocking transgenic embryos, $h s p 70: d n-f g f r 1$ and $h s p 70: c a-f g f r 1$ embryos were placed in E3 fish water in a $125-\mathrm{ml}$ flask and transferred to a $38^{\circ} \mathrm{C}$ water bath for 20 at 12,15 or $17 \mathrm{hpf}$. For double heat-shock of hsp70:gal4; UAS:myc-Notch1a-intra; hsp70:dn-fgfr1 transgenics, embryos in E3 water in a $125-\mathrm{ml}$ flask were incubated in a $38^{\circ} \mathrm{C}$ water bath for $20 \mathrm{~min}$ at $12 \mathrm{hpf}$, followed by a $45 \mathrm{~min}$ incubation in a $38^{\circ} \mathrm{C}$ water bath at 15 hpf.

WISH. Whole-mount single or double enzymatic in situ hybridization was performed on embryos fixed overnight with $4 \%$ paraformaldehyde (PFA) in phosphate buffered saline (PBS). Fixed embryos were washed briefly in PBS and transferred stepwise into methanol in PBS for storage at $-20^{\circ} \mathrm{C}$. Embryos were rehydrated stepwise through methanol in PBS-0.1\% Tween 20 (PBT). Rehydrated embryo samples were then incubated with $10 \mu \mathrm{g} \mathrm{ml}^{-1}$ proteinase $\mathrm{K}$ in PBT for $5 \mathrm{~min}$ for 5 to 10 somite stage (12-15 hpf) embryos and $15 \mathrm{~min}$ for 24 to $36 \mathrm{hpf}$ embryos. After proteinase $\mathrm{K}$ treatment, samples were washed in PBT and refixed in $4 \%$ PFA for $20 \mathrm{~min}$ at room temperature. After washes in two changes of PBT, embryos were prehybridized at $65^{\circ} \mathrm{C}$ for $1 \mathrm{~h}$ in hybridization buffer $(50 \%$ formamide, $5 \mathrm{x}$ SSC, $500 \mu \mathrm{g} \mathrm{ml}^{-1}$ torula (yeast) tRNA, $50 \mu \mathrm{g} \mathrm{ml}^{-1}$ heparin, $0.1 \%$ Tween $20,9 \mathrm{mM}$ citric acid ( $\mathrm{pH}$ 6.5)). Samples were then hybridized overnight in hybridization buffer including digoxigenin (DIG)- or fluorescein-labelled RNA probe. After hybridization, experimental samples were washed stepwise at $65^{\circ} \mathrm{C}$ for $15 \mathrm{~min}$ each in hybridization buffer in $2 \times$ SSC mix $(75 \%, 50 \%, 25 \%)$, followed by two washes with $0.2 \times \mathrm{SSC}$ for $30 \mathrm{~min}$ each at $65^{\circ} \mathrm{C}$. Further washes were performed at room temperature for $5 \mathrm{~min}$ each with $0.2 \times \mathrm{SSC}$ in PBT $(75 \%, 50 \%, 25 \%)$. Samples were incubated in PBT with $2 \%$ heat-inactivated goat serum and $2 \mathrm{mg} \mathrm{ml}^{-1}$ bovine serum albumin (block solution) for $1 \mathrm{~h}$ and then incubated overnight at $4{ }^{\circ} \mathrm{C}$ in block solution with diluted DIG-antibodies $(1: 5,000)$ conjugated with alkaline phosphatase (AP) (Roche). To visualize WISH signal, samples were washed three times in AP reaction buffer (100 mM Tris, $\mathrm{pH} 9.5,50 \mathrm{mM} \mathrm{MgCl}_{2}, 100 \mathrm{mM} \mathrm{NaCl}$, $0.1 \%$ Tween $20,1 \mathrm{mM}$ levamisole (Sigma)) for $5 \mathrm{~min}$ each and then incubated in the AP reaction buffer with NBT/BCIP substrate (Promega).

For double enzymatic WISH, the processed samples were washed in $0.1 \mathrm{M}$ glycine ( $\mathrm{pH}$ 2.2) for $20 \mathrm{~min}$ and then washed in PBT for $5 \mathrm{~min}$. After storing washed samples in methanol for $1 \mathrm{~h}$, the embryos samples were stepwise rehydrated in methanol in PBT for 5 min each $(75 \%, 50 \%, 25 \%)$ and further washed in PBT. After incubation in block solution for $1 \mathrm{~h}$, the samples were incubated overnight at $4{ }^{\circ} \mathrm{C}$ in block solution with diluted fluorescein antibodies $(1: 5,000)$ conjugated with AP (Roche). After PBT washes, the samples were washed two times in $0.1 \mathrm{M}$ Tris- $\mathrm{HCl}(\mathrm{pH}$ 8.2). Embryos were then incubated in $0.1 \mathrm{M}$ Tris- $\mathrm{HCl}$ ( $\mathrm{pH}$ 8.2) with Fast Red Tablets (Roche). After obtaining red signals, embryos were washed in $0.1 \mathrm{M}$ Tris- $\mathrm{HCl}(\mathrm{pH} 8.2)$ and refixed in $4 \%$ PFA.

For two-colour double FISH, embryos were blocked in maleic acid buffer (MAB; $150 \mathrm{mM}$ maleic acid, $100 \mathrm{mM} \mathrm{NaCl}, \mathrm{pH} 7.5$ ) with $2 \%$ Roche blocking reagent $(\mathrm{MABB})$ for $1 \mathrm{~h}$ at room temperature, after hybridizing at $65^{\circ} \mathrm{C}$ with probes as described above. Embryos were incubated overnight at $4^{\circ} \mathrm{C}$ in $\mathrm{MABB}$ with anti-fluorescein POD (Roche) at a 1:500 dilution. After four washes in MAB for $20 \mathrm{~min}$ each followed by washes in PBS at room temperature, embryo samples were incubated in TSA Plus Fluorescein Solution (Perkin Elmer) for $1 \mathrm{~h}$. Embryos were washed $10 \mathrm{~min}$ each in methanol in PBS (25\%, 50\%, 75\%, 100\%). Embryos were incubated in $1 \% \mathrm{H}_{2} \mathrm{O}_{2}$ in methanol for $30 \mathrm{~min}$ at room temperature and washed for $10 \mathrm{~min}$ each in methanol in PBS (75\%, 50\% 25\%) and $10 \mathrm{~min}$ in PBS. After blocking for $1 \mathrm{~h}$ in $\mathrm{MABB}$, embryos were incubated overnight at $4{ }^{\circ} \mathrm{C}$ in MABB with anti-DIG POD (Roche) at a 1:1,000 dilution. Samples were washed and incubated in TSA Plus CY3 solution (Perkin Elmer) as described above. Embryos were washed three times for $10 \mathrm{~min}$ each in PBT and refixed in $4 \%$ PFA after the staining was complete.

Antisense RNA probes for the following genes were prepared using probes containing DIG or fluorescein labelled UTP: runxl, cmyb, rag1, pea3, erm, scl, lmo2, $c d x 4$, shh, desma, $k d r l, c d h 5$, gata1, l-plastin, efnb2a, fli1, fgfr1, fgfr2, fgfr3, fgfr 4 , dlc, dld, dll4, notch1b, notch3, wnt16, foxc1b.

Immunofluorescence and microscopy. For immunofluorescence staining for Myc in hsp70:gal4; UAS:NICD-myc zebrafish embryos after WISH, $100 \%$ acetone was added to the embryos $\left(7 \mathrm{~min}\right.$ at $-20^{\circ} \mathrm{C}$ ) instead of proteinase $\mathrm{K}$ during the WISH procedure described above. WISH samples of embryos were blocked in MABB for $1 \mathrm{~h}$ and incubated overnight in MABB with anti-Myc monoclonal 9E10 antibodies at 1:100 (Covance). After four washes in MABB for $30 \mathrm{~min}$ each, embryos were incubated in Dylight 488 AffiniPure donkey anti-mouse IgG secondary antibodies (Jackson Immunoresearch Laboratories) at 1:100 in MABB. After staining, embryos were washed in $\mathrm{MABB}$ for four times for $30 \mathrm{~min}$ each. The samples were imaged using a stereo zoom microscope (Zeiss, Axio Zoom). Live $h s p 70: d n-f g f r 1$ and kdrl:RFP; cmy$b$ :EGFP transgenic embryos and flat-mount or whole-mount two-colour double FISH samples were imaged using confocal microscopy (Leica, SP5). GFP and fluorescein were excited by a $488-\mathrm{nm}$ laser, while DsRed and CY3 were excited by $543 \mathrm{~nm}$.

Pharmacological treatment. SU5402 (Calbiochem) and ZM306416 (Tocris) was dissolved in DMSO at a concentration of $10 \mathrm{mM}$. Zebrafish $\mathrm{AB}^{*}$ embryos were incubated in $5 \mathrm{ml}$ of $5 \mu \mathrm{M}$ SU5402 solution from 12 to $15 \mathrm{hpf}$ and $5 \mu \mathrm{M}$ ZM306416 solution from $10 \mathrm{hpf}$ in the dark, followed by fixation with $4 \%$ PFA.

Microinjections of morpholinos and mRNA. Antisense morpholinos (MOs; Gene Tools, LLC) were diluted as 1 or $3 \mathrm{mM}$ stock in DEPC-treated $\mathrm{H}_{2} \mathrm{O} .5 \mathrm{ng}$ of standard control MO (St.CoMO) ${ }^{66}$, wnt16-MO ${ }^{28}$, dld-MO2 (ref. 28), fgfr1-MO1 (ref. 67), fgfr4-MO1 ${ }^{68}$, fgfr4-CoMO2 and fgfr4-MO2 were injected at the 1- to 2-cell stage of development. The sequence of translation-blocking targeted fgfr4-MO2 is $5^{\prime}$-AGATGCTCAACATCTTGCTGAGGTA- $3^{\prime}$ and 5 base pair mismatched fgfr 4 control MO2 (fgfr4-CoMO2) is $5^{\prime}$-AaATGaTCAAaATCTT aCTGAaGTA-3' (lower case letter indicates mismatched base pairs). Full-length dlc mRNA was synthesized from linearized pCS2 $+d l c^{28}$ with the mMessege mMaching kit (Ambion). $d l c$ mRNA was injected with 100 pg into 1- to 2-cell stage $h s p 70: d n-f g f r 1$ transgenic embryos for rescue experiments. All microinjections were performed with the indicated concentration of RNA or MO in a volume of $1 \mathrm{nl}$ using a PM 1,000 cell microinjector (MDI).

FACS. fli1:EGFP and $\alpha$-actin:GFP embryos ( $\sim 50$ embryos each) at $17 \mathrm{hpf}$ were dissociated in PBS by homogenizing with sterile plastic pestle or pipette. Dissociated cells were filtered through a $40-\mu \mathrm{m}$ nylon cell strainer (Falcon 2340) and then rinsed with PBS with $1 \%$ fetal bovine serum. Propidium iodide (Sigma) was added $\left(1 \mu \mathrm{g} \mathrm{ml}^{-1}\right)$ to exclude dead cells and debris. FACS was performed based on GFP fluorescence ${ }^{5}$ with a FACS Aria II flow cytometer (Beckton Dickinson).

Quantitative real-time PCR. Total RNA was collected from posterior trunk of the embryos or whole embryos ( $\sim 30$ embryos) using TRIzol reagent (Ambion, Life Technologies) and isolated fli1:EGFP ${ }^{+}$and $\alpha$-actin:GFP ${ }^{+}$cells with the RNeasy kit (Qiagen). cDNA was generated from total RNA with iScript cDNA synthesis kit (Bio Rad). The following primers were used for cDNA quantification: ef $1 \alpha$ (forward, 5' -GAGAAGTTCGAGAAGGAAGC-3'; reverse, 5' $5^{\prime}$-CGTAGTATTTGCTGG TCTCG-3 $\left.{ }^{\prime}\right)^{33}$, runx 1 (forward, $5^{\prime}$-CGGTGAACGGTTAATATGAC-3'; reverse, $5^{\prime}$-CTTTTCATCACGGTTTATGC- $\left.3^{\prime}\right)^{33}, k d r l$ (forward, $5^{\prime}$-CTCCTGTACAGCA AGGAATG- $3^{\prime}$; reverse, $5^{\prime}$ - ATCTTTGGGCACCTTATAGC- $\left.3^{\prime}\right)^{5}$, erm (forward, $5^{\prime}$-GGTGCCTCCAAATAAGTCTC- $3^{\prime}$, reverse $5^{\prime}$ - TGGAAATCTGGAACAAA CTG-3'), efnb2a (forward, 5'-CCCATTTCCCCCAAAGACTA-3'; reverse, 5' -CTT CCCCATGAGGAGATGC-3' ${ }^{\prime}$ ), $f g f r 1$ (forward, $5^{\prime}$-AGTGATGTGGAGTTCGA GTG-3'; reverse, $5^{\prime}$-CAAGCAGGTGTACTCTCCAG- $3^{\prime}$ ) and $f g f r 4$ (forward, $5^{\prime}$-CAAACAACTTGTGGAAGAGC- ${ }^{\prime}$; reverse, $5^{\prime}$-AGCATCATGGGTAAACA CAG $\left.-3^{\prime}\right)$.

\section{References}

1. Clements, W. K. \& Traver, D. Signalling pathways that control vertebrate haematopoietic stem cell specification. Nat. Rev. Immunol. 13, 336-348 (2013).

2. McGrath, K. E. \& Palis, J. Hematopoiesis in the yolk sac: more than meets the eye. Exp. Hematol. 33, 1021-1028 (2005).

3. Medvinsky, A. L. \& Dzierzak, E. A. Development of the definitive hematopoietic hierarchy in the mouse. Dev. Comp. Immunol. 22, 289-301 (1998).

4. Cumano, A. \& Godin, I. Ontogeny of the hematopoietic system. Annu. Rev. Immunol. 25, 745-785 (2007).

5. Bertrand, J. Y. et al. Haematopoietic stem cells derive directly from aortic endothelium during development. Nature 464, 108-111 (2010).

6. Peeters, M. et al. Ventral embryonic tissues and Hedgehog proteins induce early AGM hematopoietic stem cell development. Development 136, 2613-2621 (2009).

7. Dyer, M. A., Farrington, S. M., Mohn, D., Munday, J. R. \& Baron, M. H. Indian hedgehog activates hematopoiesis and vasculogenesis and can respecify 
prospective neurectodermal cell fate in the mouse embryo. Development 128, 1717-1730 (2001).

8. Gering, M. \& Patient, R. Hedgehog signalling is required for adult blood stem cell formation in zebrafish embryos. Dev. Cell 8, 389-400 (2005).

9. Cleaver, O. \& Krieg, P. A. VEGF mediates angioblast migration during development of the dorsal aorta in Xenopus. Development 125, 3905-3914 (1998)

10. Leung, A. et al. Uncoupling VEGFA functions in arteriogenesis and hematopoietic stem cell specification. Dev. Cell 24, 144-158 (2013).

11. Shalaby, F. et al. A requirement for Flk1 in primitive and definitive hematopoiesis and vasculogenesis. Cell 89, 981-990 (1997).

12. Gupta, S., Zhu, H., Zon, L. I. \& Evans, T. BMP signaling restricts hematovascular development from lateral mesoderm during somitogenesis. Development 133, 2177-2187 (2006).

13. Maeno, M. et al. The role of BMP-4 and GATA-2 in the induction and differentiation of hematopoietic mesoderm in Xenopus laevis. Blood 88, 1965-1972 (1996).

14. Burns, C. E., Traver, D., Mayhall, E., Shepard, J. L. \& Zon, L. I. Hematopoietic stem cell fate is established by the Notch-Runx pathway. Genes Dev. 19, 2331-2342 (2005)

15. Hadland, B. K. et al. A requirement for Notch1 distinguishes 2 phases of definitive hematopoiesis during development. Blood 104, 3097-3105 (2004).

16. Kumano, K. et al. Notch1 but not Notch2 is essential for generating hematopoietic stem cells from endothelial cells. Immunity 18, 699-711 (2003).

17. Aulehla, A. \& Pourquie, O. Signaling gradients during paraxial mesoderm development. Cold Spring Harbor Perspect. Biol. 2, a000869 (2010).

18. Schier, A. F. \& Talbot, W. S. Molecular genetics of axis formation in zebrafish. Annu. Rev. Genet. 39, 561-613 (2005).

19. Murakami, M. \& Simons, M. Fibroblast growth factor regulation of neovascularization. Curr. Opin. Hematol. 15, 215-220 (2008).

20. Presta, M. et al. Fibroblast growth factor/fibroblast growth factor receptor system in angiogenesis. Cytokine. Growth. Factor. Rev. 16, 159-178 (2005).

21. Walmsley, M., Cleaver, D. \& Patient, R. Fibroblast growth factor controls the timing of Scl, Lmo2, and Runxl expression during embryonic blood development. Blood 111, 1157-1166 (2008).

22. Nakazawa, F., Nagai, H., Shin, M. \& Sheng, G. Negative regulation of primitive hematopoiesis by the FGF signaling pathway. Blood 108, 3335-3343 (2006).

23. Yamauchi, H. et al. Fgf21 is essential for haematopoiesis in zebrafish. EMBO. Rep. 7, 649-654 (2006).

24. Berardi, A. C., Wang, A., Abraham, J. \& Scadden, D. T. Basic fibroblast growth factor mediates its effects on committed myeloid progenitors by direct action and has no effect on hematopoietic stem cells. Blood 86, 2123-2129 (1995).

25. de Haan, G. et al. In vitro generation of long-term repopulating hematopoietic stem cells by fibroblast growth factor-1. Dev. Cell 4, 241-251 (2003).

26. Zhao, M. et al. FGF signaling facilitates postinjury recovery of mouse hematopoietic system. Blood 120, 1831-1842 (2012).

27. Lee, Y., Grill, S., Sanchez, A., Murphy-Ryan, M. \& Poss, K. D. Fgf signaling instructs position-dependent growth rate during zebrafish fin regeneration. Development 132, 5173-5183 (2005).

28. Clements, W. K. et al. A somitic Wnt16/Notch pathway specifies haematopoietic stem cells. Nature 474, 220-224 (2011).

29. Dequeant, M. L. et al. A complex oscillating network of signaling genes underlies the mouse segmentation clock. Science 314, 1595-1598 (2006)

30. Yamaguchi, T. P., Harpal, K., Henkemeyer, M. \& Rossant, J. fgfr-1 is required for embryonic growth and mesodermal patterning during mouse gastrulation. Genes Dev 8, 3032-3044 (1994).

31. Gering, M. \& Patient, R. Notch signalling and haematopoietic stem cell formation during embryogenesis. J. Cell Physiol. 222, 11-16 (2010).

32. Kissa, K. \& Herbomel, P. Blood stem cells emerge from aortic endothelium by a novel type of cell transition. Nature 464, 112-115 (2010).

33. Bertrand, J. Y., Kim, A. D., Teng, S. \& Traver, D. CD41 + cmyb + precursors colonize the zebrafish pronephros by a novel migration route to initiate adult hematopoiesis. Development 135, 1853-1862 (2008).

34. Munchberg, S. R., Ober, E. A. \& Steinbeisser, H. Expression of the Ets transcription factors erm and pea 3 in early zebrafish development. Mech. Dev. 88, 233-236 (1999).

35. Roehl, H. \& Nusslein-Volhard, C. Zebrafish pea3 and erm are general targets of FGF8 signaling. Curr. Biol. 11, 503-507 (2001).

36. Patterson, L. J. et al. The transcription factors Scl and Lmo2 act together during development of the hemangioblast in zebrafish. Blood 109, 2389-2398 (2007).

37. Ren, X., Gomez, G. A., Zhang, B. \& Lin, S. Scl isoforms act downstream of etsrp to specify angioblasts and definitive hematopoietic stem cells. Blood 115, 5338-5346 (2010).

38. Zhong, T. P., Childs, S., Leu, J. P. \& Fishman, M. C. Gridlock signalling pathway fashions the first embryonic artery. Nature 414, 216-220 (2001).

39. Goldfarb, M. Signaling by fibroblast growth factors: the inside story. Science's STKE: Signal Transduction Knowledge Environ. 2001, pe37 (2001).
40. Marques, S. R., Lee, Y., Poss, K. D. \& Yelon, D. Reiterative roles for FGF signaling in the establishment of size and proportion of the zebrafish heart. Dev. Biol. 321, 397-406 (2008).

41. Lee, H. C., Tseng, W. A., Lo, F. Y., Liu, T. M. \& Tsai, H. J. FoxD5 mediates anterior-posterior polarity through upstream modulator Fgf signaling during zebrafish somitogenesis. Dev. Biol. 336, 232-245 (2009).

42. Scheer, N. \& Campos-Ortega, J. A. Use of the Gal4-UAS technique for targeted gene expression in the zebrafish. Mech. Dev. 80, 153-158 (1999).

43. Thisse, B., Thisse, C. \& Weston, J. A. Novel FGF receptor (Z-FGFR4) is dynamically expressed in mesoderm and neurectoderm during early zebrafish embryogenesis. Dev. Dynamics 203, 377-391 (1995).

44. Amaya, E., Musci, T. J. \& Kirschner, M. W. Expression of a dominant negative mutant of the FGF receptor disrupts mesoderm formation in Xenopus embryos. Cell 66, 257-270 (1991).

45. Griffin, K., Patient, R. \& Holder, N. Analysis of FGF function in normal and no tail zebrafish embryos reveals separate mechanisms for formation of the trunk and the tail. Development 121, 2983-2994 (1995).

46. Pouget, C. et al. FGF signalling restricts haematopoietic stem cell specification via modulation of the BMP pathway. Nat Commun. doi:10.1038/ncomms6588.

47. Lawson, N. D., Vogel, A. M. \& Weinstein, B. M. Sonic hedgehog and vascular endothelial growth factor act upstream of the Notch pathway during arterial endothelial differentiation. Dev. Cell 3, 127-136 (2002).

48. Rowlinson, J. M. \& Gering, M. Hey2 acts upstream of Notch in hematopoietic stem cell specification in zebrafish embryos. Blood 116, 2046-2056 (2010).

49. Songhet, P., Adzic, D., Reibe, S. \& Rohr, K. B. fgf1 is required for normal differentiation of erythrocytes in zebrafish primitive hematopoiesis. Dev. Dynamics 236, 633-643 (2007).

50. Reifers, F. et al. Fgf8 is mutated in zebrafish acerebellar (ace) mutants and is required for maintenance of midbrain-hindbrain boundary development and somitogenesis. Development 125, 2381-2395 (1998).

51. Cao, Y. et al. fgf17b, a novel member of Fgf family, helps patterning zebrafish embryos. Dev. Biol. 271, 130-143 (2004).

52. Draper, B. W., Stock, D. W. \& Kimmel, C. B. Zebrafish fgf24 functions with fgf8 to promote posterior mesodermal development. Development 130, 4639-4654 (2003).

53. Pourquie, O. Vertebrate segmentation: from cyclic gene networks to scoliosis. Cell 145, 650-663 (2011).

54. Pouget, C., Pottin, K. \& Jaffredo, T. Sclerotomal origin of vascular smooth muscle cells and pericytes in the embryo. Dev. Biol. 315, 437-447 (2008).

55. Wasteson, P. et al. Developmental origin of smooth muscle cells in the descending aorta in mice. Development 135, 1823-1832 (2008).

56. Wiegreffe, C., Christ, B., Huang, R. \& Scaal, M. Sclerotomal origin of smooth muscle cells in the wall of the avian dorsal aorta. Dev. Dynamics 236, 2578-2585 (2007).

57. Wiegreffe, C., Christ, B., Huang, R. \& Scaal, M. Remodeling of aortic smooth muscle during avian embryonic development. Dev. Dynamics 238, 624-631 (2009).

58. Esner, M. et al. Smooth muscle of the dorsal aorta shares a common clonal origin with skeletal muscle of the myotome. Development 133, 737-749 (2006).

59. Kobayashi, I. et al. Jam1a-Jam2a interactions regulate haematopoietic stem cell fate through Notch signalling. Nature 512, 319-323 (2014).

60. Nguyen, P. D. et al. Haematopoietic stem cell induction by somite-derived endothelial cells controlled by meox1. Nature 512, 314-318 (2014).

61. Pouget, C., Gautier, R., Teillet, M. A. \& Jaffredo, T. Somite-derived cells replace ventral aortic hemangioblasts and provide aortic smooth muscle cells of the trunk. Development 133, 1013-1022 (2006).

62. North, T. E. et al. Prostaglandin E2 regulates vertebrate haematopoietic stem cell homeostasis. Nature 447, 1007-1011 (2007).

63. Huang, H., Zhang, B., Hartenstein, P. A., Chen, J. N. \& Lin, S. NXT2 is required for embryonic heart development in zebrafish. BMC. Dev. Biol. 5, 7 (2005).

64. Lawson, N. D. \& Weinstein, B. M. In vivo imaging of embryonic vascular development using transgenic zebrafish. Dev. Biol. 248, 307-318 (2002).

65. Higashijima, S., Okamoto, H., Ueno, N., Hotta, Y. \& Eguchi, G. High-frequency generation of transgenic zebrafish which reliably express GFP in whole muscles or the whole body by using promoters of zebrafish origin. Dev. Biol. 192, 289-299 (1997).

66. Stachura, D. L. et al. The zebrafish granulocyte colony-stimulating factors (Gcsfs): 2 paralogous cytokines and their roles in hematopoietic development and maintenance. Blood 122, 3918-3928 (2013).

67. Scholpp, S., Groth, C., Lohs, C., Lardelli, M. \& Brand, M. Zebrafish fgfr1 is a member of the fgf8 synexpression group and is required for fgf8 signalling at the midbrain-hindbrain boundary. Dev. Genes Evol. 214, 285-295 (2004).

68. Nakayama, Y. et al. Fgf19 is required for zebrafish lens and retina development. Dev. Biol. 313, 752-766 (2008).

\section{Acknowledgements}

We thank K.D. Poss for sharing transgenic animals and in situ probes,

D. Yelon for sharing imaging equipment, and K. Ong and R. Rainville for 
animal care. Y.L. was supported by a postdoctoral fellowship from the American Heart Association. This work was supported by Innovative Science Award \#12PILT12860010 from the American Heart Association, R01-DK074482 and R01-HL119056 from the National Institutes of Health (D.T.).

\section{Author contributions}

Y.L. designed the experiments, D.T. supervised the experiments.,Y.L., J.E.M. and A.D.K. performed experiments, Y.L., C.P. and D.L.D analysed the data and, Y.L., W.K.C. and D.T. wrote the manuscript

\section{Additional information}

Supplementary Information accompanies this paper at http://www.nature.com/ naturecommunications

Competing financial interests: The authors declare no competing financial interests.

Reprints and permission information is available online at http://npg.nature.com/ reprintsandpermissions/

How to cite this article: Lee, Y. et al. FGF signalling specifies haematopoietic stem cells through its regulation of somitic Notch signalling. Nat. Commun. 5:5583 doi: $10.1038 /$ ncomms6583 (2014). 